Article

\title{
Towards Zn-Dominant Tourmaline: A Case of Zn-Rich Fluor-Elbaite and Elbaite from the Julianna System at Piława Górna, Lower Silesia, SW Poland
}

\author{
Adam Pieczka ${ }^{1, *}$, Bożena Gołębiowska ${ }^{1}$, Piotr Jeleń ${ }^{2}$ (D), Adam Włodek ${ }^{1}$, Eligiusz Szełęg ${ }^{3}$ \\ and Adam Szuszkiewicz 4 (iD) \\ 1 Department of Mineralogy, Petrography and Geochemistry, AGH University of Science and Technology, \\ Mickiewicza 30, 30-059 Kraków, Poland; goleb@agh.edu.pl (B.G.); wlodek@agh.edu.pl (A.W.) \\ 2 Faculty of Materials Science and Ceramics, AGH University of Science and Technology, Mickiewicza 30, \\ 30-059 Kraków, Poland; pjelen@agh.edu.pl \\ 3 Faculty of Earth Sciences, University of Silesia in Katowice, Będzińska 60, 41-200 Sosnowiec, Poland; \\ eligiusz.szeleg@us.edu.pl \\ 4 Institute of Geological Sciences, University of Wrocław, pl. M. Borna 9, 50-204 Wrocław, Poland; \\ adam.szuszkiewicz@uwr.edu.pl \\ * Correspondence: pieczka@agh.edu.pl; Tel.: +48-12-617-2144
}

Received: 18 January 2018; Accepted: 16 March 2018; Published: 22 March 2018

\begin{abstract}
Tourmalines are a group of minerals which may concentrate various accessory components, e.g., $\mathrm{Cu}, \mathrm{Ni}, \mathrm{Zn}, \mathrm{Bi}, \mathrm{Ti}$, and $\mathrm{Sn}$. The paper presents fluor-elbaite and elbaite from a dyke of the Julianna pegmatitic system at Piława Górna, at the NE margin of the Bohemian Massif, SW Poland, containing up to 6.32 and $7.37 \mathrm{wt} \% \mathrm{ZnO}$, respectively. Such high amounts of $\mathrm{ZnO}$ are almost two times higher than in the second most Zn-enriched tourmaline known to date. The compositions of the Zn-rich tourmalines from Piława Górna, studied by electron micropropy and Raman spectroscopy, correspond to the formulae: ${ }^{X}\left(\mathrm{Na}_{0.733} \mathrm{Ca}_{0.013} \square_{0.254}\right)_{\Sigma 1}{ }^{\mathrm{Y}}\left(\mathrm{Al}_{1.033} \mathrm{Li}_{0.792}\right.$ $\left.\mathrm{Zn}_{0.755} \mathrm{Fe}^{2+}{ }_{0.326} \mathrm{Mn}_{0.094}\right)_{\Sigma 3} \mathrm{Z} \mathrm{Al}_{6}\left({ }^{\mathrm{T}} \mathrm{Si}_{6} \mathrm{O}_{18}\right)\left(\mathrm{BO}_{3}\right)_{3}{ }^{\mathrm{V}}(\mathrm{OH})_{3}{ }^{\mathrm{W}}\left(\mathrm{F}_{0.654} \mathrm{OH}_{0.344}\right)$, and $\mathrm{X}^{\mathrm{X}}\left(\mathrm{Na}_{0.779} \mathrm{Ca}_{0.015} \square_{0.206}\right)_{\Sigma 1}$ $\mathrm{Y}\left(\mathrm{Al}_{1.061} \mathrm{Li}_{0.869} \mathrm{Zn}_{0.880} \mathrm{Fe}^{2+}{ }_{0.098} \mathrm{Mn}_{0.094}\right)_{\Sigma 3} \mathrm{Z}^{\mathrm{Z}} \mathrm{Al}_{6}\left({ }^{\mathrm{T}} \mathrm{Si}_{6} \mathrm{O}_{18}\right)\left(\mathrm{BO}_{3}\right)_{3}{ }^{\mathrm{V}}(\mathrm{OH})_{3}{ }^{\mathrm{W}}\left(\mathrm{OH}_{0.837} \mathrm{~F}_{0.163}\right)$, respectively, with $\mathrm{Zn}$ as one of the main octahedral occupants. A comparison with other tourmalines and associated $\mathrm{Zn}$-rich fluor-elbaite and elbaite from the pegmatite indicates that atypically high $\mathrm{Zn}$-enrichment is not a result of $\mathrm{Zn}$-Fe fractionation, but dissolution and reprecipitation induced by a late $(\mathrm{Na}, \mathrm{Li}, \mathrm{B}, \mathrm{F})$-bearing fluid within the assemblage of gahnite spinel and primary schorl-type tourmaline. This strongly suggests Na-Li-B-F metasomatism of gahnite-bearing mineral assemblages as that is the only environment that can promote crystallization of a hypothetical Zn-dominant tourmaline. The compositions of the $\mathrm{Zn}$-rich fluor-elbaite and elbaite suggest three possible end-members for such a hypothetical tourmaline species: $\mathrm{NaZn}_{3} \mathrm{Al}_{6}\left(\mathrm{Si}_{6} \mathrm{O}_{18}\right)\left(\mathrm{BO}_{3}\right)_{3}(\mathrm{OH})_{3}(\mathrm{OH})$, $\square\left(\mathrm{Zn}_{2} \mathrm{Al}\right) \mathrm{Al}_{6}\left(\mathrm{Si}_{6} \mathrm{O}_{18}\right)\left(\mathrm{BO}_{3}\right)_{3}(\mathrm{OH})_{3}(\mathrm{OH})$ and $\mathrm{Na}\left(\mathrm{Zn}_{2} \mathrm{Al}\right) \mathrm{Al}_{6}\left(\mathrm{Si}_{6} \mathrm{O}_{18}\right)\left(\mathrm{BO}_{3}\right)_{3}(\mathrm{OH})_{3} \mathrm{O}$ by analogy with other tourmalines with divalent $\mathrm{Y}$ occupants, such as schorl/foitite/oxy-schorl and dravite/magnesio-foitite/oxy-dravite.
\end{abstract}

Keywords: tourmaline; fluor-elbaite; elbaite; Zn-enrichment; electron microprobe; Raman spectroscopy; granitic pegmatites; Piława Górna; Sudetes

\section{Introduction}

Tourmalines are a supergroup of accessory minerals, common in various igneous, metamorphic and sedimentary rocks. They are complex borosilicates with the generalized chemical formula 
$\mathrm{XY}_{3} \mathrm{Z}_{6}\left(\mathrm{~T}_{6} \mathrm{O}_{18}\right)\left(\mathrm{BO}_{3}\right)_{3} \mathrm{~V}_{3} \mathrm{~W}$ [1], where $\mathrm{X}, \mathrm{Y}, \mathrm{Z}, \mathrm{T}, \mathrm{B}, \mathrm{V}(=\mathrm{O} 3)$ and $\mathrm{W}(=\mathrm{O} 1)$ denote structural sites occupied by:

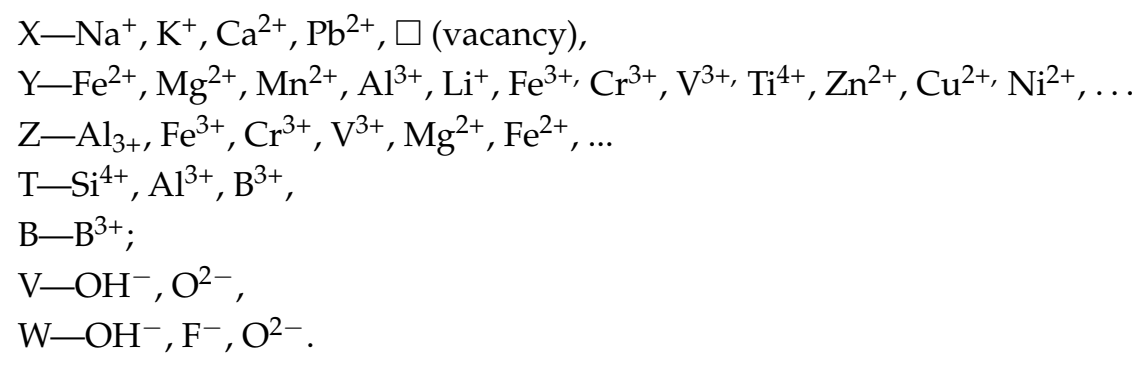

An extremely complex chemical composition results in a number of minerals accepted as valid species by the Commission on New Minerals, Nomenclature and Classification of the International Mineralogical Association (IMA CNMNC), or reported but not IMA-approved, e.g., an unnamed $\mathrm{Pb}$-dominant tourmaline (up to $17.5 \mathrm{wt} \% \mathrm{PbO}$; [2]). Varieties atypically enriched in other elements, which do not attain the status of the dominant component in any structural site in any tourmaline species, are also occasionally found. For example, apart from the relatively common $\left(\mathrm{Fe}^{2+}, \mathrm{Mg}^{2+}, \mathrm{Mn}^{2+}\right.$, $\mathrm{Al}^{3+}, \mathrm{Al}^{3+}+\mathrm{Li}^{+}, \mathrm{Fe}^{3+}, \mathrm{Cr}^{3+}$ or $\left.\mathrm{V}^{3+}\right)$-dominant tourmalines, there are also known crystals enriched in $\mathrm{Ti}^{4+}$ (up to $4.07 \mathrm{wt} \% \mathrm{TiO}_{2} ;[3]$ ), $\mathrm{Sn}^{4+}$ (up to $0.42 \mathrm{wt} \% \mathrm{SnO}_{2} ;[4]$ ), $\mathrm{Bi}^{3+}$ (up to 0.49 wt $\% \mathrm{Bi}_{2} \mathrm{O}_{3} ;$ [5]), $\mathrm{Cu}^{2+}$ (up to $3.51 \mathrm{wt} \% \mathrm{CuO}$; [6]), $\mathrm{Ni}^{2+}$ (up to $3.96 \mathrm{wt} \% \mathrm{NiO}$; [7]), and $\mathrm{Zn}^{2+}$. Other admixtures may also be present although they do not attain such spectacular concentrations. Exceptional diversity of chemical composition results in numerous color varieties, and the most beautiful tourmaline crystals are precious gems. In geological sciences, tourmalines are highly treasured as records of a signature of the conditions and chemistry of their environments due to their (1) widespread occurrence and stability over a significant portion of the pressure-temperature-composition space of the crust; (2) accommodation of exceptional range of geochemically different elements; (3) response and reflection of physical and chemical formation conditions by their trace element and isotopic compositions and of element source-reservoirs; and (4) negligible intracrystalline element diffusion over geologic time, even at high temperatures [8].

Zinc is found in numerous tourmalines, although commonly at amounts not exceeding a few tenths of wt \%. Rare findings of tourmalines with $\mathrm{ZnO}>1 \mathrm{wt} \%$ have been reported, for instance, from Cross Lake, MB, Canada (1.24 wt \%; [9]), Minas Gerais (1.72 wt \%; [10]; $1.88 \mathrm{wt} \mathrm{\% ;} \mathrm{[11])} \mathrm{and} \mathrm{Rio} \mathrm{Grande}$ do Norte (2.15 wt \%; [12]; $2.43 \mathrm{wt} \%$; [13]; $3.01 \mathrm{wt} \%$; [14]) in Brazil, and from Congo (2.85 wt \%; [15]). The most $\mathrm{Zn}$-enriched tourmaline (3.83 wt \% $\mathrm{ZnO}$ ) has been described from Russia [16].

In this paper, we describe fluor-elbaite and elbaite with almost $7.5 \mathrm{wt} \% \mathrm{ZnO}$ from the Julianna system of anatectic pegmatites at Piława Górna, Góry Sowie Block, SW Poland. We discuss their chemistry and Raman spectra especially in the range of $\mathrm{OH}$ vibrations, propose possible explanations for such high $\mathrm{Zn}$ enrichment and suggest the most probable environments for formation of even more Zn-rich crystals.

\section{Geological Setting}

Zinc-enriched tourmaline was found in a pegmatite of the Julianna pegmatitic system exposed in an amphibolite-migmatite quarry $\left(50^{\circ} 42^{\prime} 11.77^{\prime \prime} \mathrm{N}, 16^{\circ} 44^{\prime} 12.36^{\prime \prime}\right.$ E) near Piława Górna, $\sim 50$ km southwest of Wrocław, SW Poland (Figures 1 and 2). The quarry is located in the Góry Sowie Block (GSB), a tectono-stratigraphic unit that is mostly built of a polymetamorphic metasedimentary-metavolcanic sequence and is situated at the north-eastern periphery of the Bohemian Massif in the European Variscides. The Piława Górna quarry exploits the migmatite-amphibolite rock deposit with over 320 millions Mt of estimated resources for crushing rocks aggregates. Migmatite accounts for about $72 \%$ of the raw rock and the rest is mainly amphibolite, with minor bodies of granitic pegmatite. The Julianna pegmatitic system is composed of coeval and cogenetic dykes, apophyses and lenticular bodies, up to 6-7 $\mathrm{m}$ thick, extending along $30-40 \mathrm{~m}$ in vertical section, $80-100 \mathrm{~m}$ in planar view 
along a NNE-SSW running zone of strongly tectonized amphibolite [17]. The pegmatites show a broad range of textural differentiation from homogeneous and subhomogeneous to simply zoned bodies (border zone + wall zone + graphic intermediate zone + blocky-feldspar intermediate zone + quartz core \pm quartz-albite zone \pm spodumene-“lepidolite” core). Geochemical variability varies from primitive and moderately fractionated pegmatites, enriched in $\mathrm{Nb}-\mathrm{REE}-\mathrm{Be}-\mathrm{B}$ and belonging to the NYF (niobium-yttrium-fluorine) pegmatitic family, to rare and much more strongly fractionated pods located in the axial parts of the largest dykes that contain Li-Cs-Ta-Be-B mineralization of the LCT (lithium-cesium-tantalum) type [17-20]. The pegmatites are formed mainly of microcline, Na-plagioclase, quartz, "biotite" and muscovite, accompanied by schorl, almandine-spessartine garnet and beryl. Rare-element mineralization of the prevalent NYF-affiliated pegmatites includes, among others, columbite-group minerals, ixiolite, ferrowodginite, samarskite-, euxenite- and fergusonite-group minerals, pyrochlore-supergroup minerals, cassiterite, ilmenite and titanite, gadolinite-group minerals, hellandite-( $(Y)$, keiviite-(Y), pilawite-(Y), allanite-group minerals, xenotime-(Y), and monazite-(Ce). Highly fractionated pods with the LCT-type mineralization contain, among others, "zinnwaldite", "lepidolite", a Cs-bearing dark mica, spodumene, pollucite, cassiterite, spessartine, tantalite-(Mn), minerals of the microlite group, elbaite-liddicoatite-rossmanite tourmaline, Cs-bearing beryl and pezzottaite. Mineralogy and petrography of the Julianna pegmatites have been systematically studied $[17,19,21-23]$ and the occurrence is the type locality for pilawite-(Y), bohseite and żabińskiite [24-26].

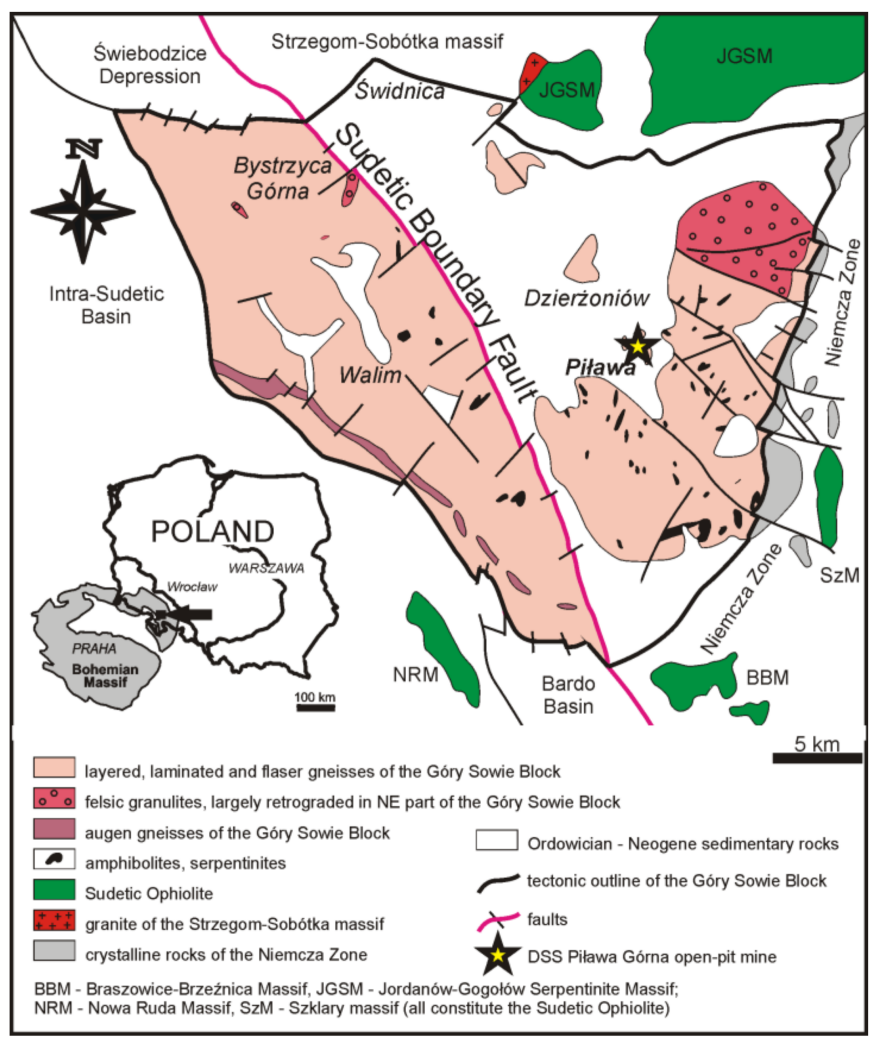

Figure 1. Simplified geological map of the Góry Sowie Block with the location of the Piława Górna quarry (modified after Szuszkiewicz et al. [17]).

Dating of Julianna pegmatites gave an emplacement age of $377.6 \pm 1.3 \mathrm{Ma}$ [U-Th-Pb; monazite-(Ce)] and $380.7 \pm 2.4 \mathrm{Ma}$ (U-Pb-Th; uraninite) $[20,27]$. These ages point to their formation by the anatectic melting of the metasedimentary-metavolcanic GSB rocks during tectonic exhumation at 385-370 Ma [28-30]. Recent studies of trace elements in quartz from the Julianna pegmatites and their host rocks comply with this model and suggest that the pegmatite-forming melt was most probably 
generated at pressures $\sim 500 \mathrm{MPa}$, at a slightly greater depth than the present day exposure level [31]. The geochemical diversity of the metasedimentary-metavolcanic protolith, similar to paragneisses and amphibolites exposed in the Piława Górna quarry, is a plausible source for highly enriched partial melts with hybrid NYF + LCT characteristics.

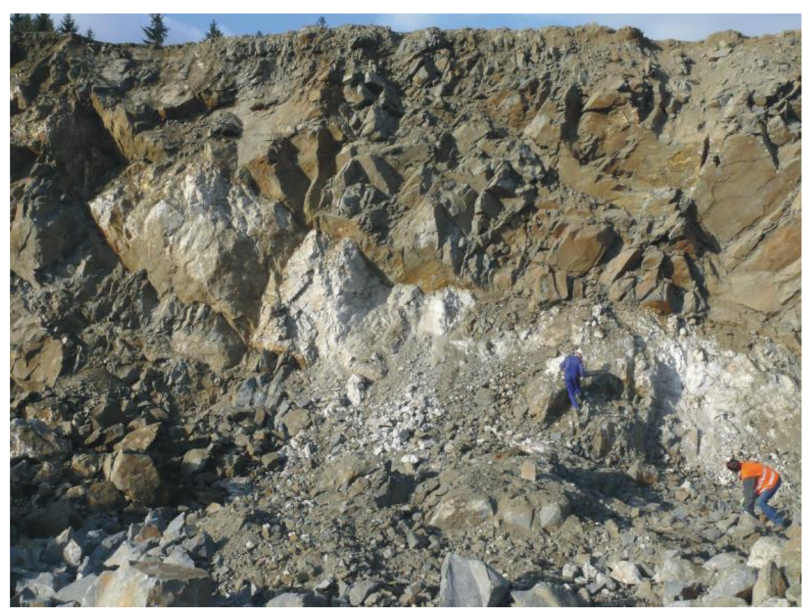

Figure 2. A fragment of the Julianna pegmatitic system in a migmatite-amphibolite quarry at Piława Górna; (year 2010).

\section{Occurrence}

The pegmatite dyke with the Zn-rich tourmaline was exposed in the Piława Górna quarry in 2010, when it could be observed along a few tens of meters in horizontal section with a maximum thickness of $\sim 3-4 \mathrm{~m}$. The dyke showed typical zoning, and the assemblage of accessory minerals locates it as transitional between NYF- and LCT-type pegmatites. Fan-shaped aggregates of muscovite, a few centimeters in length, in the graphic zone are a characteristic feature of this pegmatite. Short prismatic crystals of light-grey-bluish beryl, up to $5 \mathrm{~cm}$ long, occur together with quartz in interstices among feldspar crystals in the blocky feldspar zone and in the quartz core [22]. The blocky feldspar zone and the quartz core contain also up to $7 \mathrm{~cm}$ long intergrowths and radial aggregates of black tourmaline, up to 4-5 cm large quartz-garnet (almandine-spessartine series) symplectitic intergrowths, a few centimeter sized books of pale greenish muscovite, relatively abundant cassiterite (up to $3 \mathrm{~cm}$ large) and columbite-group minerals (up to $4 \mathrm{~cm}$ in length), as well as subordinate, a few millimeter sized dark greenish crystals of gahnite. Lithium-bearing tourmalines were found only occasionally in the form of small, up to $2 \mathrm{~cm}$ long, dark green crystals within the books of greenish K-mica. Electron microprobe studies of the collected material reveal also the presence of columbite-(Fe) and columbite-(Mn), ferrowodginite and wodginite, various pyrochlore-supergroup minerals (fluorcalciomicrolite, hydroxylcalciomicrolite or kenocalciomicrolite, hydroxylplumbomicrolite or oxyplumbomicrolite, hydromicrolite and hydropyrochlore grading to zero-valence-dominant microlite and pyrochlore, and fluorcalcioroméite), Fe-bearing Cs-dominant dark mica, genthelvite, uraninite, monazite-(Ce), xenotime-(Y), cheralite, zircon, scheelite, sphalerite, ferronigerite and zinconigerite, lithiophilite, alluaudite, hydroxylapatite, mitridatite and other unrecognized phosphates, pucherite, and probably also cookeite and chamosite. Zinc-rich tourmaline was found only along the contact between adjacent pale greenish, transparent gahnite and primary schorl-type tourmaline evolving to secondary fluor-elbaite. This Zn-rich tourmaline, like other Li-tourmalines of the pegmatite system at Piława Górna, has no gemological value not only due to its very small dimensions and exceptional rarity, but mainly due to numerous inclusions and general nontransparency. Its pale greenish color originates from the presence of traces of $\mathrm{Fe}^{2+}$ and $\mathrm{Mn}^{2+}$, whereas $\mathrm{Zn}^{2+}$ as an element of the first transition series with electron configuration [Ar] $3 \mathrm{~d}^{10} 4 \mathrm{~s}^{2}$ is not a coloring agent. 


\section{Methods}

\subsection{Electron Microprobe Analysis (EMPA)}

Electron microprobe analyses of tourmalines and other rare minerals of the pegmatite were performed at the Inter-Institute Analytical Complex for Minerals and Synthetic Substances at the University of Warsaw, Poland, using a Cameca SX 100 electron microprobe (CAMECA, Cedex, France). Samples were prepared as 1-inch discs containing small fragments of the pegmatite with crystals designed for analyses, with grinded and polished surface. Polished surfaces of the discs were coated with carbon. Zinc-rich tourmaline was found during analyzing crystals of transparent, pale greenish gahnite associated with dark tourmaline. The microprobe operated in wavelength-dispersive mode under the following conditions: accelerating voltage of $15 \mathrm{kV}$, beam current of $10 \mathrm{nA}$, beam diameter of $2 \mu \mathrm{m}$, peak count-time of $20 \mathrm{~s}$, background time of $10 \mathrm{~s}$. Standards, diffracting crystals, analytical lines and mean detection limits (in wt \%) were as follows: fluorophlogopite-F (PC0, K $\alpha, 0.12)$, albite- $-\mathrm{Na}$ (TAP, $K \alpha, 0.03$ ), diopside-Mg (TAP, $K \alpha, 0.02)$, Si (TAP, $K \alpha, 0.03)$ and Ca (PET, $K \alpha, 0.02)$, orthoclase-Al (TAP, $K \alpha, 0.03)$ and $\mathrm{K}(\mathrm{PET}, K \alpha, 0.02)$, rutile-Ti (LPET, $K \alpha, 0.02)$, rhodonite-Mn (LIF, $K \alpha, 0.09)$, hematite-Fe (LIF, $K \alpha, 0.08), \mathrm{V}_{2} \mathrm{O}_{5}-\mathrm{V}(\mathrm{LIF}, K \alpha, 0.06), \mathrm{Cr}_{2} \mathrm{O}_{3}-\mathrm{Cr}(\mathrm{LPET}, K \alpha, 0.02)$, and sphalerite-Zn (LIF, $K \alpha, 0.09)$. The raw data were reduced with the PAP routine of Pouchou and Pichoir [32].

Atomic contents were normalized to $15 \mathrm{Y}+\mathrm{Z}+\mathrm{T}$ atoms per formula unit (apfu) for schorl-type tourmalines with $\mathrm{Y}+\mathrm{Z}>9$ apfu $\left(\mathrm{Li}_{\text {calc. }}=0\right.$ apfu), and on the basis of $6 \mathrm{Si}$ apfu for Li-bearing tourmalines, with $\mathrm{B}_{2} \mathrm{O}_{3}$ calculated with the assumption of $3 \mathrm{~B}$ apfu, $\mathrm{Li}=9-(\mathrm{Y}+\mathrm{Z})_{\mathrm{EMPA}}$ apfu, where $(Y+Z)_{\text {EMPA }}$ denotes the total of all octahedral $Y$ - and Z-site occupants determined by EMPA, $\mathrm{Fe}_{\text {total }}=\mathrm{Fe}^{2+}$, and $\mathrm{H}_{2} \mathrm{O}$ occurring as $\mathrm{OH}$ groups calculated by stoichiometry based on electroneutrality of the formulae. For analyses showing $\mathrm{OH}+\mathrm{F}>4$ apfu due to excesses of microprobe determined $\mathrm{SiO}_{2}$ (quartz nanoinclusions), the amount of the component was reduced to a value for which the stoichiometric $\mathrm{OH}+\mathrm{F}=4$ apfu was achieved, i.e., the highest content accepted for the tourmaline structure. The estimation of the $\mathrm{Li}_{2} \mathrm{O}$ amounts from EMPA on the basis of a multiple regression equation given by Pesquera et al. [33] is not applicable in our case, because the calculated $\mathrm{Li}_{2} \mathrm{O}$ amount seems to be $\mathrm{ZnO}$ dependent for high $\mathrm{ZnO}$ contents (Figure 3). Comparing the contents of $\mathrm{Li}_{2} \mathrm{O}$ obtained from our calculation procedure and the amounts from the regression equation by Pesquera et al. [33], all data points corresponding to Zn-rich tourmaline deviate distinctly from the 1:1 trend and, in our opinion it is due to high contents of $\mathrm{ZnO}$, a component which was not included as an important independent variable in the multiple regression by Pesquera et al. [33].

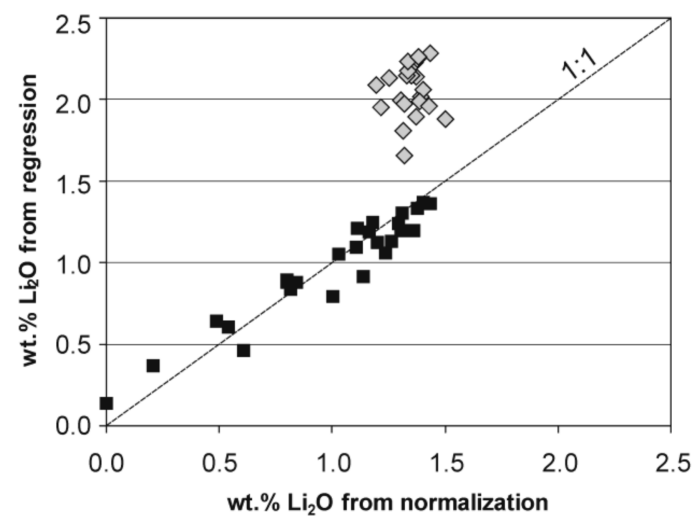

Figure 3. Covariation between wt $\% \mathrm{Li}_{2} \mathrm{O}$ of fluor-elbaite and elbaite calculated on the basis of the used normalization vs. amounts calculated from the regression equation of Pesquera et al. [33]. Symbols: dark squares-analyses with $\mathrm{ZnO}<1.20 \mathrm{wt} \%$, grey diamonds-analyses with $\mathrm{ZnO}>3.50 \mathrm{wt} \%$. 


\subsection{Raman Spectroscopy}

Raman spectra were collected in back-scattered geometry at the Faculty of Materials Science and Ceramics, AGH UST, Cracow, Poland, with a Horiba Labram HR spectrometer integrated with an Olympus BX 40 confocal microscope equipped with a Nd: YAG, $532 \mathrm{~nm}(10 \mathrm{~mW})$ laser and $1800 \mathrm{~g} / \mathrm{mm}$ grating. The spectra were recorded in the range $4000-50 \mathrm{~cm}^{-1}$ on randomly oriented surfaces of crystals mounted in epoxy resin in a 1-inch disc that was used also for EMPA studies. The Raman measurements were carried out with an estimated analytical spot size of $\sim 1 \mu \mathrm{m}$, the microscope magnification $100 \times$, an acquisition time of $600 \mathrm{~s}$ and accumulation of 2 scans. Calibration was done using the $520.7 \mathrm{~cm}^{-1}$ line of $\mathrm{Si}$, and for deconvolution of the recorded spectra we used the commercially available program SPECTRA-CALC ${ }^{\mathrm{TM}}$ (sc) [34].

\section{Results}

\subsection{Primary Tourmalines}

The only tourmalines discernible by naked eye in hand specimens are a few centimeters large, black, and sometimes radially intergrown crystals of primary schorl (Tur I; the used abbreviations for mineral names according to Whitney and Evans [35]). The crystals display almost constant FeO contents [13.27(30)-12.66(29) wt \%; 1.88(4)-1.81(4) Fe apfu on average] and small Si deficiency [5.97(2) and 5.94(3) Si apfu, respectively] (Table 1). However, they show some heterogeneity in other chemical components as well as some Mn-Fe fractionation. The most primitive variety is Tur IA, with $\mathrm{Mn} /(\mathrm{Mn}+\mathrm{Fe})=0.010(2)$, and depletion in $\mathrm{Al}$ [6.14(6) apfu on average]. More evolved, is Tur IB that show $\mathrm{Mn} /(\mathrm{Mn}+\mathrm{Fe})=0.029(14)-0.034(4)$, and much higher Al content of 6.79(11)-6.91(4) apfu. Compared to Tur IB, Tur IA is also enriched in Na [0.66(3) vs. 0.58(3)-0.54(5) apfu] and Mg [0.81(6) vs. 0.26(11)-0.23(5) apfu], and shows higher contents of such trace components as Ca [0.09(2) vs. 0.01(1) $a p f u]$ and $\mathrm{Ti}[0.14(4)$ vs. 0.011(4) apfu], and lower amounts of Mn [0.020(4) vs. 0.056(26) apfu] and Zn $[0.009(8)$ vs. 0.038(12) apfu]. Fluorine contents are almost the same in Tur IA and Tur IB [0.08(3) vs. $0.07(4)-0.12(2) a p f u$, respectively] and the $W$ site in both types of schorl is dominated by monovalent anions $\left(\mathrm{OH}^{-}+\mathrm{F}^{-}\right)$with $\mathrm{OH}^{-}>\mathrm{F}^{-}$, with slightly increasing ${ }^{\mathrm{W}} \mathrm{O}^{2-}$ in the more evolved variety [0.24(5) vs. 0.36(9) apfu in Tur IA and Tur IB, respectively].

\subsection{Secondary Dark Bluish Li-Bearing Tourmaline in Quartz}

Anhedral inclusions ( $<1 \mathrm{~mm}$ ) of dark bluish Li-bearing tourmaline (Tur IIA) in quartz, replacing schorl and fluor-schorl, are discernible under a stereomicroscope or in back-scattered-electron (BSE) images. The schorl-type primary tourmaline is slightly enriched in $\mathrm{Li}_{2} \mathrm{O}$ (up to $\sim 0.61 \mathrm{wt} \%$; $0.404 \mathrm{Li}$ apfu) (Table 2; anal. 223/9 and 217/3). However, low contents of Ca (0.006-0.021 apfu) and Ti (up to $0.019 a p f u$ ), depletion in Fe down to $1.400 a p f u$, enrichment in Al (6.834-7.030 apfu), Mn (0.098-0.173 apfu) and Zn (0.059-0.069 apfu; 0.49-0.56 wt \% ZnO), as well as the degree of Mn-Fe fractionation $[\mathrm{Mn} /(\mathrm{Mn}+\mathrm{Fe})=0.066-0.103]$ indicate that it corresponds to the primary Al-bearing Tur IB. The ${ }^{\mathrm{W}} \mathrm{O}^{2-}$ content in this tourmaline reaches 0.46 apfu.

Secondary Tur IIA represents elbaite and fluor-elbaite with rather constant Na (0.721-0.802 apfu). Compared to its Tur IB precursor, it has generally higher, although partly overlapping $\mathrm{Mn} /(\mathrm{Mn}+\mathrm{Fe})$ ratio of $0.079-0.183$, similar or slightly higher contents of $\mathrm{Al}$ (up to 7.069 apfu) and Mn (0.091-0.170 apfu), a noticeable increase of $\mathrm{Zn}(0.044-0.141$ apfu; up to $1.17 \mathrm{wt} \% \mathrm{ZnO}$ ), decrease of Fe (down to 0.704 apfu) and constant low contents of Ca (0.026-0.043 apfu), Mg (up to 0.011 apfu) and Ti (up to 0.010 apfu). Lithium can increase up to 0.941 apfu and F up to 0.676 apfu. 
Table 1. Average compositions of primary schorl-type tourmaline.

\begin{tabular}{|c|c|c|c|}
\hline \multirow{2}{*}{$a p f u$} & \multirow{2}{*}{$\begin{array}{c}\text { Tur IA Sample } 224 \\
\qquad n=13\end{array}$} & \multicolumn{2}{|c|}{ Tur IB Sample 226} \\
\hline & & $n=13$ & $n=54$ \\
\hline $\mathrm{SiO}_{2}$ & $35.21(25)$ & $35.11(27)$ & $34.84(28)$ \\
\hline $\mathrm{TiO}_{2}$ & $1.11(30)$ & $0.09(3)$ & $0.10(3)$ \\
\hline $\mathrm{B}_{2} \mathrm{O}_{3 \text { (calc.) }}$ & $10.25(6)$ & $10.26(4)$ & $10.19(6)$ \\
\hline $\mathrm{Al}_{2} \mathrm{O}_{3}$ & $30.86(38)$ & $34.28(48)$ & $34.38(32)$ \\
\hline $\mathrm{FeO}$ & $13.27(30)$ & $13.04(31)$ & $12.66(29)$ \\
\hline $\mathrm{MnO}$ & $0.14(3)$ & $0.39(18)$ & $0.45(6)$ \\
\hline $\mathrm{MgO}$ & $3.19(23)$ & $1.03(43)$ & $0.89(18)$ \\
\hline $\mathrm{ZnO}$ & $0.07(06)$ & $0.30(10)$ & $0.27(4)$ \\
\hline $\mathrm{CaO}$ & $0.48(10)$ & $0.06(6)$ & $0.03(2)$ \\
\hline $\mathrm{Na}_{2} \mathrm{O}$ & 2.01(10) & $1.77(10)$ & $1.64(14)$ \\
\hline $\mathrm{K}_{2} \mathrm{O}$ & $0.04(2)$ & $0.03(1)$ & $0.04(1)$ \\
\hline $\mathrm{H}_{2} \mathrm{O}$ & $3.25(6)$ & $3.16(11)$ & $3.07(5)$ \\
\hline $\mathrm{F}_{2}$ & $0.14(7)$ & $0.14(8)$ & $0.23(3)$ \\
\hline$-\mathrm{O}=\mathrm{F}_{2}$ & $-0.06(3)$ & $-0.06(4)$ & $-0.10(1)$ \\
\hline Total & $99.96(56)$ & $99.60(44)$ & $98.68(59)$ \\
\hline \multicolumn{4}{|l|}{$a p f u$} \\
\hline $\mathrm{X}_{\mathrm{Na}^{+}}$ & $0.660(33)$ & $0.582(33)$ & $0.544(48)$ \\
\hline $\mathrm{X}_{\mathrm{K}^{+}}$ & $0.009(4)$ & $0.006(2)$ & $0.008(1)$ \\
\hline${ }^{X} \mathrm{Ca}^{2+}$ & $0.087(18)$ & $0.010(11)$ & $0.006(3)$ \\
\hline $\mathrm{x}_{\square}$ & $0.244(42)$ & $0.402(40)$ & $0.443(49)$ \\
\hline$\Sigma X$ & 1.000 & 1.000 & 1.000 \\
\hline $\mathrm{Mg}^{2+}$ & $0.806(57)$ & $0.261(107)$ & $0.227(46)$ \\
\hline $\mathrm{Fe}^{2+}$ & $1.882(43)$ & $1.847(41)$ & $1.807(43)$ \\
\hline $\mathrm{Mn}^{2+}$ & $0.020(4)$ & $0.056(26)$ & $0.064(8)$ \\
\hline $\mathrm{Zn}^{2+}$ & $0.009(8)$ & $0.038(12)$ & $0.034(5)$ \\
\hline $\mathrm{Al}^{3+}$ & $6.142(63)$ & $6.787(108)$ & 6.912(37) \\
\hline $\mathrm{Ti}^{4+}$ & $0.142(39)$ & $0.011(4)$ & $0.013(4)$ \\
\hline$\Sigma(\mathrm{Y}+\mathrm{Z})$ & 9.000 & 9.000 & 9.000 \\
\hline${ }^{\mathrm{B}} \mathrm{B}^{3+}$ & 3.000 & 3.000 & 3.000 \\
\hline${ }^{\mathrm{T}} \mathrm{Si}^{4+}$ & $5.973(22)$ & $5.945(36)$ & $5.943(34)$ \\
\hline${ }^{\mathrm{T}} \mathrm{Al}^{3+}$ & $0.027(22)$ & $0.055(36)$ & $0.057(34)$ \\
\hline$\Sigma \mathrm{T}$ & 6.000 & 6.000 & 6.000 \\
\hline $\mathrm{O}$ & 27.000 & 27.000 & 27.000 \\
\hline${ }^{\mathrm{v}_{\mathrm{OH}}}{ }^{-}$ & 3.000 & 3.000 & 3.000 \\
\hline${ }^{\mathrm{W}} \mathrm{O}^{2-}$ & $0.241(53)$ & $0.363(85)$ & $0.386(51)$ \\
\hline${ }^{\mathrm{W}} \mathrm{OH}^{-}$ & $0.683(62)$ & $0.565(117)$ & $0.491(51)$ \\
\hline $\mathrm{W}_{\mathrm{F}^{-}}$ & $0.076(35)$ & $0.073(45)$ & $0.123(16)$ \\
\hline$\Sigma W$ & 1.000 & 1.000 & 1.000 \\
\hline $\mathrm{Mn \#}$ & $0.010(2)$ & $0.029(14)$ & $0.034(4)$ \\
\hline $\mathrm{Zn \#}$ & $0.005(4)$ & $0.020(6)$ & $0.019(3)$ \\
\hline Zn1\# & $0.011(2)$ & $0.043(17)$ & $0.046(4)$ \\
\hline
\end{tabular}

$\mathrm{Mn} \#=\mathrm{Mn} /(\mathrm{Mn}+\mathrm{Fe}) ; \mathrm{Zn} \#=\mathrm{Zn} /(\mathrm{Zn}+\mathrm{Fe}) ; \mathrm{Zn} 1 \#=(\mathrm{Zn}+\mathrm{Mn}) /(\mathrm{Zn}+\mathrm{Mn}+\mathrm{Fe}+\mathrm{Mg}) ; n-\mathrm{number}$ of analyses; calc.-calculate. Data in parentheses denote standard deviations.

\subsection{Secondary Dark Greenish Fluor-Elbaite in Muscovite Books}

Dark greenish translucent Li-tourmaline (Tur IIB) is extremely rare, although relatively large, with euhedral crystals being up to $2 \mathrm{~cm}$ in length and $0.5 \mathrm{~cm}$ in diameter. In spite of the differences in color and size, the crystals are compositionally close to Tur IIA, except that the most Fe-enriched domains with 8.24-7.39 wt \% FeO (1.141-1.013 Fe apfu) are even more Al-enriched (up to 7.293 apfu) (Table 2). With increasing Mn-Fe fractionation $[\mathrm{Mn} /(\mathrm{Mn}+\mathrm{Fe})=0.082-0.161]$, Tur IIB shows a decrease of Fe down to 0.684 apfu, coupled with an increase of $\mathrm{Al}$ and $\mathrm{Mn}$ up to 7.315 apfu and 0.131 apfu, respectively. Simultaneously, the calculated Li content increases from 0.526 apfu to 
$0.875 a p f u$. The amounts of $\mathrm{Mg}$ and Ti are negligible, 0.014-0.038 apfu and 0.006-0.012 apfu, respectively. The contents of $\mathrm{Zn}$ vary randomly (0.13-0.93 wt \% ZnO; up to $0.111 \mathrm{Zn} a p f u)$, although generally, they increase in sectors with higher Mn-Fe fractionation degrees. With fluorine contents from 0.382 up to 0.591 apfu and ${ }^{\mathrm{W}} \mathrm{OH}^{-}<<\mathrm{W}_{\mathrm{F}}{ }^{-}$, Tur IIB classifies as fluor-elbaite. Only several Al-poorest and Fe-richest compositions, with lowest measured $\mathrm{F}$ and calculated $\mathrm{Li}$ contents and ${ }^{\mathrm{W}} \mathrm{O}^{2-}>{ }^{\mathrm{W}} \mathrm{F}^{-}+{ }^{\mathrm{W}} \mathrm{OH}^{-}$, might be classified as Fe-bearing darrelhenryite [the end-member composition $\mathrm{Na}\left(\mathrm{LiAl}_{2}\right) \mathrm{Al}_{6} \mathrm{~B}_{3} \mathrm{Si}_{6} \mathrm{O}_{27}(\mathrm{OH})_{3} \mathrm{O}$ ].

Table 2. Representative compositions of dark bluish (Tur IIA) and dark green (Tur IIB) tourmaline.

\begin{tabular}{|c|c|c|c|c|c|c|c|c|c|c|}
\hline \multirow{2}{*}{ wt $\%$} & \multicolumn{5}{|c|}{ Tur IIA } & \multicolumn{5}{|c|}{ Tur IIB } \\
\hline & $223 / 9$ & $217 / 3$ & $217 / 4$ & $217 / 6$ & $217 / 5$ & $112 / 28$ & $112 / 23$ & $112 / 16$ & $112 / 17$ & $112 / 20$ \\
\hline $\mathrm{SiO}_{2}$ & 36.36 & 35.68 & 37.00 & 36.77 & 36.73 & 36.25 & 36.64 & 37.03 & 37.13 & 37.45 \\
\hline $\mathrm{TiO}_{2}$ & 0.03 & 0.15 & 0.07 & 0.07 & 0.02 & 0.19 & 0.07 & 0.08 & 0.08 & 0.08 \\
\hline $\mathrm{B}_{2} \mathrm{O}_{3 \text { (calc.) }}$ & 10.53 & 10.34 & 10.72 & 10.65 & 10.64 & 10.50 & 10.61 & 10.73 & 10.76 & 10.85 \\
\hline $\mathrm{Al}_{2} \mathrm{O}_{3}$ & 35.14 & 35.47 & 36.12 & 36.71 & 36.64 & 36.60 & 37.79 & 37.97 & 38.09 & 38.74 \\
\hline $\mathrm{FeO}$ & 10.96 & 9.95 & 8.64 & 5.44 & 5.52 & 8.24 & 7.49 & 6.53 & 5.67 & 5.10 \\
\hline $\mathrm{MnO}$ & 1.24 & 0.69 & 0.73 & 0.90 & 0.99 & 0.73 & 0.69 & 0.85 & 0.82 & 0.97 \\
\hline $\mathrm{MgO}$ & 0.05 & 0.07 & 0.08 & 0.03 & 0.02 & 0.13 & 0.13 & 0.10 & 0.11 & 0.13 \\
\hline $\mathrm{ZnO}$ & 0.49 & 0.56 & 0.49 & 1.03 & 1.17 & 0.17 & 0.13 & 0.38 & 0.93 & 0.57 \\
\hline $\mathrm{CaO}$ & 0.03 & 0.12 & 0.18 & 0.17 & 0.15 & 0.60 & 0.07 & 0.10 & 0.12 & 0.15 \\
\hline $\mathrm{Li}_{2} \mathrm{O}_{\text {(calc.) }}$ & 0.61 & 0.54 & 1.14 & 1.43 & 1.38 & 0.82 & 0.80 & 1.03 & 1.11 & 1.18 \\
\hline $\mathrm{Na}_{2} \mathrm{O}$ & 1.74 & 2.17 & 2.38 & 2.54 & 2.51 & 2.28 & 2.35 & 2.52 & 2.41 & 2.47 \\
\hline $\mathrm{K}_{2} \mathrm{O}$ & 0.02 & 0.04 & 0.05 & 0.02 & 0.00 & 0.03 & 0.02 & 0.00 & 0.03 & 0.00 \\
\hline $\mathrm{H}_{2} \mathrm{O}_{\text {(calc.) }}$ & 3.49 & 2.87 & 3.32 & 3.28 & 3.08 & 2.74 & 2.80 & 2.82 & 2.89 & 2.95 \\
\hline $\mathrm{F}_{2}$ & 0.27 & 0.61 & 0.80 & 0.83 & 1.25 & 0.73 & 0.75 & 1.03 & 1.05 & 0.90 \\
\hline$-\mathrm{O}=\mathrm{F}_{2}$ & -0.12 & -0.26 & -0.34 & -0.35 & -0.53 & -0.31 & -0.32 & -0.43 & -0.44 & -0.38 \\
\hline Total & 100.85 & 99.00 & 101.37 & 99.53 & 99.58 & 99.69 & 100.04 & 100.73 & 100.74 & 101.15 \\
\hline \multicolumn{11}{|l|}{$a p f u$} \\
\hline $\mathrm{x}_{\mathrm{Na}^{+}}$ & 0.558 & 0.708 & 0.750 & 0.802 & 0.794 & 0.731 & 0.746 & 0.793 & 0.753 & 0.767 \\
\hline $\mathrm{x}_{\mathrm{K}^{+}}$ & 0.005 & 0.008 & 0.010 & 0.004 & 0.000 & 0.006 & 0.004 & 0.000 & 0.006 & 0.000 \\
\hline${ }^{x} \mathrm{Ca}^{2+}$ & 0.006 & 0.021 & 0.031 & 0.029 & 0.026 & 0.106 & 0.012 & 0.018 & 0.020 & 0.025 \\
\hline$x_{\square}$ & 0.431 & 0.264 & 0.209 & 0.165 & 0.180 & 0.157 & 0.239 & 0.189 & 0.220 & 0.207 \\
\hline$\Sigma \bar{X}$ & 1.000 & 1.000 & 1.000 & 1.000 & 1.000 & 1.000 & 1.000 & 1.000 & 1.000 & 1.000 \\
\hline $\mathrm{Li}^{+}$ & 0.404 & 0.365 & 0.741 & 0.941 & 0.906 & 0.543 & 0.528 & 0.670 & 0.721 & 0.761 \\
\hline $\mathrm{Mg}^{2+}$ & 0.013 & 0.018 & 0.018 & 0.000 & 0.005 & 0.031 & 0.031 & 0.024 & 0.026 & 0.031 \\
\hline $\mathrm{Fe}^{2+}$ & 1.513 & 1.400 & 1.171 & 0.742 & 0.754 & 1.141 & 1.026 & 0.885 & 0.767 & 0.684 \\
\hline $\mathrm{Mn}^{2+}$ & 0.173 & 0.098 & 0.101 & 0.124 & 0.137 & 0.102 & 0.096 & 0.116 & 0.112 & 0.131 \\
\hline $\mathrm{Zn}^{2+}$ & 0.059 & 0.069 & 0.058 & 0.124 & 0.141 & 0.021 & 0.016 & 0.045 & 0.111 & 0.067 \\
\hline $\mathrm{Al}^{3+}$ & 6.834 & 7.030 & 6.902 & 7.060 & 7.054 & 7.139 & 7.293 & 7.250 & 7.253 & 7.315 \\
\hline $\mathrm{Ti}^{4+}$ & 0.003 & 0.019 & 0.008 & 0.009 & 0.003 & 0.024 & 0.009 & 0.010 & 0.010 & 0.010 \\
\hline$\Sigma(Y+Z)$ & 9.000 & 9.000 & 9.000 & 9.000 & 9.000 & 9.000 & 9.000 & 9.000 & 9.000 & 9.000 \\
\hline${ }^{\mathrm{B}} \mathrm{B}^{3+}$ & 3.000 & 3.000 & 3.000 & 3.000 & 3.000 & 3.000 & 3.000 & 3.000 & 3.000 & 3.000 \\
\hline${ }^{\mathrm{T}} \mathrm{Si}^{4+}$ & 6.000 & 6.000 & 6.000 & 6.000 & 6.000 & 6.000 & 6.000 & 6.000 & 6.000 & 6.000 \\
\hline $\mathrm{O}$ & 27.000 & 27.000 & 27.000 & 27.000 & 27.000 & 27.000 & 27.000 & 27.000 & 27.000 & 27.000 \\
\hline${ }^{\mathrm{v}} \mathrm{OH}^{-}$ & 3.000 & 3.000 & 3.000 & 3.000 & 3.000 & 3.000 & 3.000 & 3.000 & 3.000 & 3.000 \\
\hline${ }^{\mathrm{W}} \mathrm{O}^{2-}$ & 0.011 & 0.462 & 0.000 & 0.000 & 0.000 & 0.593 & 0.555 & 0.429 & 0.351 & 0.392 \\
\hline${ }^{\mathrm{w}} \mathrm{OH}^{-}$ & 0.846 & 0.214 & 0.589 & 0.570 & 0.355 & 0.024 & 0.054 & 0.044 & 0.113 & 0.153 \\
\hline $\mathrm{w}_{\mathrm{F}^{-}}$ & 0.143 & 0.324 & 0.411 & 0.430 & 0.645 & 0.382 & 0.391 & 0.527 & 0.536 & 0.455 \\
\hline$\Sigma \mathrm{W}$ & 1.000 & 1.000 & 1.000 & 1.000 & 1.000 & 1.000 & 1.000 & 1.000 & 1.000 & 1.000 \\
\hline Mn\# & 0.103 & 0.066 & 0.079 & 0.143 & 0.154 & 0.082 & 0.086 & 0.116 & 0.127 & 0.161 \\
\hline Zn\# & 0.038 & 0.047 & 0.047 & 0.143 & 0.158 & 0.018 & 0.016 & 0.048 & 0.127 & 0.089 \\
\hline \multirow[t]{2}{*}{ Zn1\# } & 0.132 & 0.106 & 0.118 & 0.251 & 0.268 & 0.095 & 0.096 & 0.151 & 0.220 & 0.217 \\
\hline & Sch & F-sch & Elb & Elb & F-elb & $\mathrm{Dh}$ & $\mathrm{Dh}$ & F-elb & F-elb & F-elb \\
\hline
\end{tabular}

$\mathrm{Mn} \#=\mathrm{Mn} /(\mathrm{Mn}+\mathrm{Fe}) ; \mathrm{Zn} \#$ = Zn/(Zn + Fe); Zn1\# = (Zn + Mn)/(Zn + Mn + Fe + Mg); Abbreviations: Sch—schorl, F-sch—fluor-schorl, Elb—elbaite, Dh—darrelhenryite, F-elb—fluor-elbaite; 223/9-sample ID/analysis number, calc.-calculate. 


\subsection{Zn-Rich Tourmaline from Gahnite Dissolution-Fluor-Elbaite + Elbaite Reprecipitation Zones}

Zn-rich tourmaline (Tur III) was found as a secondary phase in a metasomatically altered assemblage of adjacent Tur IB (oxy-schorl to fluor-schorl) and gahnite. Highly fractionated pale greenish gahnite with composition $\mathrm{Zn}\left(\mathrm{Al}_{1.98} \mathrm{Fe}^{3+}{ }_{0.02}\right) \mathrm{O}_{4}[\mathrm{Zn} /(\mathrm{Zn}+\mathrm{Fe})=0.965(3)]$, typically occurs as fractured crystals, up to a few hundred micrometers in size, that are partly overgrown by Tur III (Figure 4). Tur IB is usually largely dissolved and replaced by Tur IIIA (Zn-rich fluor-elbaite), forming clearly zoned crystals up to 1 millimeter in size (Figure 4). Compositional maps (Figure 5) present elemental distribution of $\mathrm{Fe}, \mathrm{Mn}, \mathrm{Zn}$ and $\mathrm{Al}$ in two areas, in which $\mathrm{Zn}$-enriched tourmaline was detected. In both cases, Fe concentration decreases gradually, coupled with increasing $\mathrm{Zn}$ and $\mathrm{Al}$ from the center of the zoned crystals outwards (i.e., from Tur IB to Tur IIIA) and towards the adjacent gahnite. A local increase in Mn content in Tur IIIA is also marked. Tur IIIB (Zn-rich elbaite) occurs only as tiny domains, with the the highest $\mathrm{Zn}$ concentration marked in Figure 5 (Zn) as yellowish points within the greenish matrix of Tur IIIA. The Zn-rich fluor-elbaite (Tur IIIA) and elbaite (Tur IIIB) appear only along the gahnite margins as a discontinuous zone with a maximum thickness of $\sim 100 \mu \mathrm{m}$. Secondary Tur III sometimes contains relics of the primary Tur IB and numerous inclusions of mainly quartz and albite, sometimes gahnite, and very rarely sphalerite. Table 3 presents representative analyses of primary tourmaline and both secondary Zn-rich tourmalines. The chemical compositions of Tur IB in the gahnite-tourmaline assemblage do not differ from typical compositions of primary schorl Tur IB (Table 1). Tur IIIA corresponds to fluor-elbaite with 0.535-0.658 apfu F and contains 6.993-7.051 apfu Al, 0.256-0.567 apfu Fe and 0.082-0.113 apfu Mn. Tur IIIB, on the other hand, classifies as elbaite with 0.163-0.388 apfu F, and has slightly higher contents of Al (7.042-7.150 apfu), and similar amounts of Fe (0.058-0.198 apfu) and Mn (0.054-0.097 apfu). Magnesium and Ti are below detection limits in both secondary tourmalines.
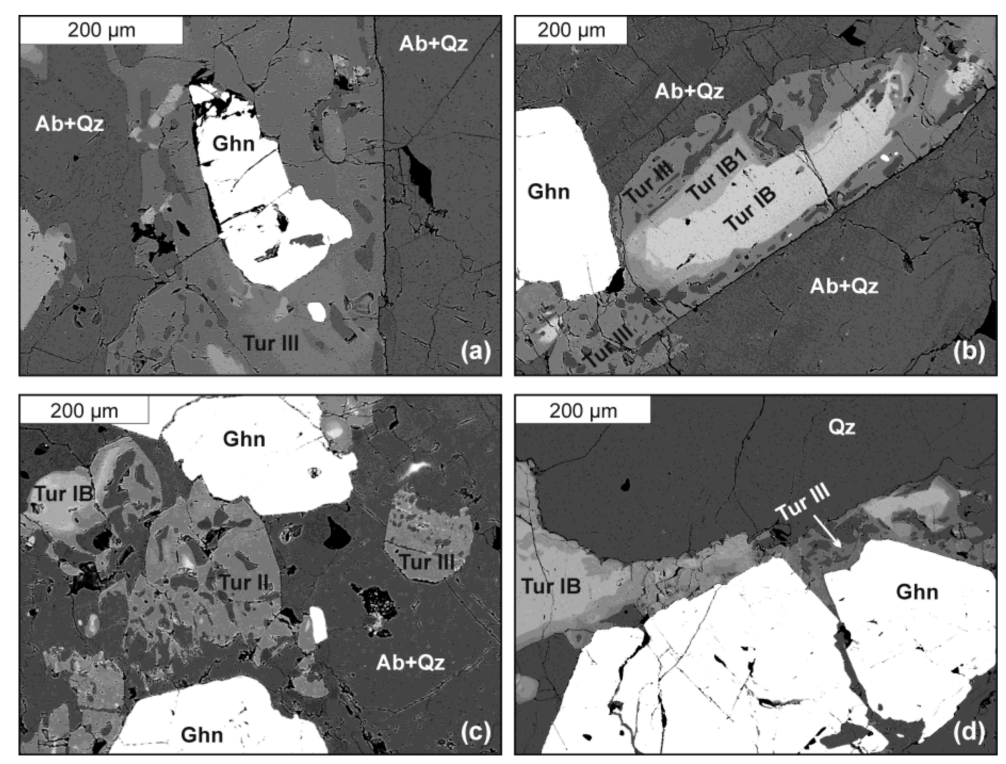

Figure 4. Back-scattered-electron images of Zn-rich tourmaline (Tur III): (a) Tur III overgrowing gahnite; (b,c) Tur III overgrowing primary schorl-type Tur IB and Tur IB1; (d) a zone of Tur III around gahnite. Abbreviations: Ab-albite, Qz-quartz, Ghn-gahnite, Tur IB-primary, schorl-type tourmaline, Tur IB1-fluor-schorl, Tur III-Zn-rich fluor-elbaite. 
Table 3. Representative compositions of tourmalines of the Zn-rich assemblage.

\begin{tabular}{|c|c|c|c|c|c|c|c|c|c|c|}
\hline \multirow{2}{*}{ wt $\%$} & \multirow{2}{*}{$\begin{array}{r}\text { Tur IB } \\
225 / 1\end{array}$} & \multirow{2}{*}{$\begin{array}{r}\text { Tur IB1 } \\
225 / 2\end{array}$} & \multicolumn{3}{|c|}{ Tur IIIA } & \multicolumn{5}{|c|}{ Tur IIIB } \\
\hline & & & $225 / 3$ & $225 / 4$ & $225 / 5$ & $225 / 6$ & $225 / 7$ & $225 / 8$ & $225 / 9$ & $225 / 10$ \\
\hline $\mathrm{SiO}_{2}$ & 34.96 & 35.83 & 36.73 & 37.08 & 37.03 & 37.05 & 36.61 & 37.25 & 36.79 & 37.11 \\
\hline $\mathrm{TiO}_{2}$ & 0.18 & 0.06 & 0.03 & 0.00 & 0.00 & 0.00 & 0.00 & 0.00 & 0.00 & 0.00 \\
\hline $\mathrm{B}_{2} \mathrm{O}_{3 \text { (calc.) }}$ & 10.13 & 10.38 & 10.64 & 10.74 & 10.73 & 10.73 & 10.61 & 10.79 & 10.66 & 10.75 \\
\hline $\mathrm{Al}_{2} \mathrm{O}_{3}$ & 33.57 & 35.08 & 36.48 & 36.98 & 36.83 & 37.17 & 36.86 & 37.67 & 36.99 & 37.05 \\
\hline $\mathrm{FeO}$ & 13.27 & 9.76 & 3.21 & 1.92 & 2.40 & 1.26 & 1.17 & 0.62 & 0.51 & 0.71 \\
\hline $\mathrm{MnO}$ & 0.82 & 1.06 & 0.72 & 0.76 & 0.68 & 0.52 & 0.60 & 0.48 & 0.54 & 0.69 \\
\hline $\mathrm{MgO}$ & 0.18 & 0.06 & 0.00 & 0.00 & 0.00 & 0.00 & 0.00 & 0.00 & 0.00 & 0.00 \\
\hline $\mathrm{ZnO}$ & 0.93 & 1.66 & 4.73 & 5.68 & 6.32 & 6.67 & 6.72 & 6.87 & 6.97 & 7.37 \\
\hline $\mathrm{CaO}$ & 0.03 & 0.06 & 0.10 & 0.10 & 0.08 & 0.02 & 0.04 & 0.02 & 0.02 & 0.08 \\
\hline $\mathrm{Li}_{2} \mathrm{O}_{\text {(calc.) }}$ & 0.00 & 0.49 & 1.32 & 1.39 & 1.22 & 1.33 & 1.25 & 1.37 & 1.38 & 1.34 \\
\hline $\mathrm{Na}_{2} \mathrm{O}$ & 1.99 & 2.15 & 2.52 & 2.62 & 2.33 & 2.43 & 2.16 & 2.33 & 2.50 & 2.49 \\
\hline $\mathrm{K}_{2} \mathrm{O}$ & 0.03 & 0.00 & 0.00 & 0.00 & 0.00 & 0.00 & 0.02 & 0.00 & 0.00 & 0.00 \\
\hline $\mathrm{H}_{2} \mathrm{O}_{\text {(calc.) }}$ & 2.80 & 2.97 & 3.12 & 3.14 & 3.09 & 3.39 & 3.33 & 3.50 & 3.48 & 3.56 \\
\hline $\mathrm{F}_{2}$ & 0.52 & 0.68 & 1.17 & 1.19 & 1.28 & 0.65 & 0.69 & 0.47 & 0.42 & 0.32 \\
\hline$-\mathrm{O}=\mathrm{F}_{2}$ & -0.22 & -0.29 & -0.49 & -0.50 & -0.54 & -0.28 & -0.29 & -0.20 & -0.18 & -0.13 \\
\hline Total & 99.19 & 99.95 & 100.26 & 101.10 & 101.45 & 100.96 & 99.77 & 101.16 & 100.08 & 101.32 \\
\hline \multicolumn{11}{|l|}{$a p f u$} \\
\hline${ }^{\mathrm{X}} \mathrm{Na}^{+}$ & 0.664 & 0.698 & 0.799 & 0.822 & 0.733 & 0.764 & 0.688 & 0.727 & 0.791 & 0.779 \\
\hline $\mathrm{x}_{\mathrm{K}^{+}}$ & 0.007 & 0.000 & 0.000 & 0.000 & 0.000 & 0.000 & 0.004 & 0.000 & 0.000 & 0.000 \\
\hline${ }^{X} \mathrm{Ca}^{2+}$ & 0.005 & 0.011 & 0.017 & 0.016 & 0.013 & 0.004 & 0.006 & 0.003 & 0.003 & 0.015 \\
\hline $\mathrm{x}_{\square}$ & 0.324 & 0.292 & 0.184 & 0.162 & 0.254 & 0.232 & 0.302 & 0.269 & 0.206 & 0.206 \\
\hline$\Sigma X$ & 1.000 & 1.000 & 1.000 & 1.000 & 1.000 & 1.000 & 1.000 & 1.000 & 1.000 & 1.000 \\
\hline $\mathrm{Li}^{+}$ & 0.000 & 0.331 & 0.865 & 0.906 & 0.792 & 0.867 & 0.824 & 0.884 & 0.906 & 0.869 \\
\hline $\mathrm{Mg}^{2+}$ & 0.047 & 0.015 & 0.000 & 0.000 & 0.000 & 0.000 & 0.000 & 0.000 & 0.000 & 0.000 \\
\hline $\mathrm{Fe}^{2+}$ & 1.904 & 1.367 & 0.439 & 0.260 & 0.326 & 0.170 & 0.161 & 0.084 & 0.075 & 0.098 \\
\hline $\mathrm{Mn}^{2+}$ & 0.120 & 0.151 & 0.099 & 0.104 & 0.094 & 0.071 & 0.083 & 0.065 & 0.070 & 0.094 \\
\hline $\mathrm{Zn}^{2+}$ & 0.117 & 0.205 & 0.570 & 0.679 & 0.755 & 0.798 & 0.813 & 0.817 & 0.840 & 0.880 \\
\hline $\mathrm{Al}^{3+}$ & 6.789 & 6.924 & 7.023 & 7.051 & 7.033 & 7.095 & 7.119 & 7.150 & 7.109 & 7.061 \\
\hline $\mathrm{Ti}^{4+}$ & 0.023 & 0.007 & 0.004 & 0.000 & 0.000 & 0.000 & 0.000 & 0.000 & 0.000 & 0.000 \\
\hline$\Sigma(\mathrm{Y}+\mathrm{Z})$ & 9.000 & 9.000 & 9.000 & 9.000 & 9.000 & 9.000 & 9.000 & 9.000 & 9.000 & 9.000 \\
\hline${ }^{B} B^{3+}$ & 3.000 & 3.000 & 3.000 & 3.000 & 3.000 & 3.000 & 3.000 & 3.000 & 3.000 & 3.000 \\
\hline${ }^{\mathrm{T}} \mathrm{Si}^{4+}$ & 6.000 & 6.000 & 6.000 & 6.000 & 6.000 & 6.000 & 6.000 & 6.000 & 6.000 & 6.000 \\
\hline $\mathrm{O}$ & 27.000 & 27.000 & 27.000 & 27.000 & 27.000 & 27.000 & 27.000 & 27.000 & 27.000 & 27.000 \\
\hline${ }^{\mathrm{v}} \mathrm{OH}^{-}$ & 3.000 & 3.000 & 3.000 & 3.000 & 3.000 & 3.000 & 3.000 & 3.000 & 3.000 & 3.000 \\
\hline${ }^{\mathrm{W}} \mathrm{O}^{2-}$ & 0.515 & 0.327 & 0.000 & 0.000 & 0.000 & 0.000 & 0.000 & 0.000 & 0.000 & 0.000 \\
\hline${ }^{\mathrm{W}} \mathrm{OH}^{-}$ & 0.201 & 0.313 & 0.398 & 0.389 & 0.344 & 0.665 & 0.643 & 0.759 & 0.785 & 0.837 \\
\hline $\mathrm{w}_{\mathrm{F}^{-}}$ & 0.284 & 0.360 & 0.602 & 0.611 & 0.656 & 0.335 & 0.357 & 0.241 & 0.215 & 0.163 \\
\hline$\Sigma \mathrm{W}$ & 1.000 & 1.000 & 1.000 & 1.000 & 1.000 & 1.000 & 1.000 & 1.000 & 1.000 & 1.000 \\
\hline Mn\# & 0.059 & 0.099 & 0.184 & 0.286 & 0.223 & 0.293 & 0.340 & 0.439 & 0.517 & 0.495 \\
\hline Zn\# & 0.058 & 0.130 & 0.565 & 0.723 & 0.699 & 0.824 & 0.835 & 0.907 & 0.923 & 0.901 \\
\hline \multirow[t]{2}{*}{ Zn1\# } & 0.108 & 0.205 & 0.604 & 0.751 & 0.723 & 0.836 & 0.848 & 0.913 & 0.929 & 0.910 \\
\hline & O-sch & F-sch & F-elb & F-elb & F-elb & Elb & $\mathrm{Elb}$ & Elb & Elb & $\mathrm{Elb}$ \\
\hline
\end{tabular}

$\mathrm{Mn} \#$ = Mn/(Mn + Fe); Zn\# = Zn/(Zn + Fe); Zn1\# = (Zn + Mn)/(Zn + Mn + Fe + Mg). Abbreviations: O-sch-oxy-schorl, F-sch—fluor-schorl, F-elb—fluor-elbaite, Elb—elbaite. 225/1—sample ID/analysis number, calc.—calculate. 

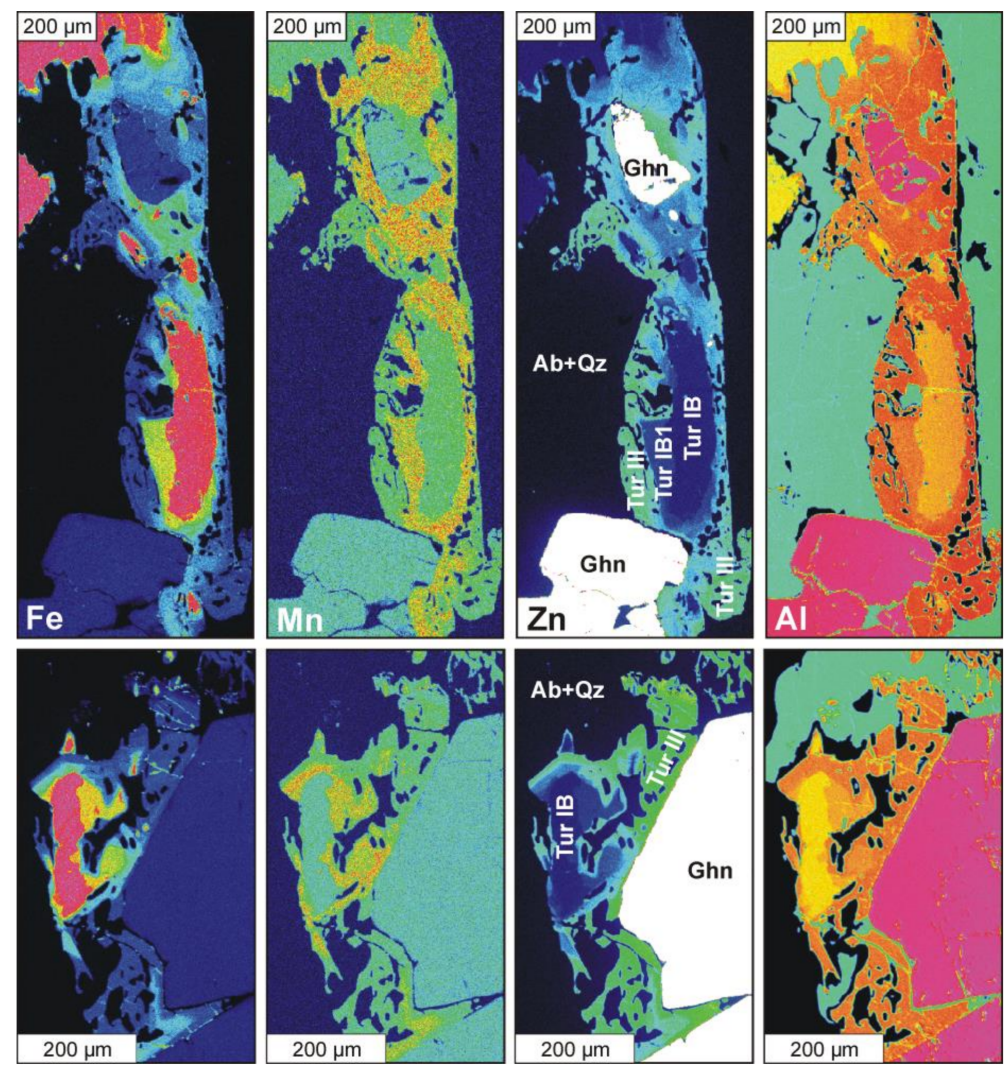

Figure 5. Fe-, Mn-, Zn- and Al-mapping for two occurrences of Zn-rich tourmaline (compare with Figure 3). Abbreviations of mineral names as in Figure 4. Color scale of increasing concentrations of the elements: dark blue-blue-green-yellow-orange-red-purple-white.

The content of $\mathrm{Zn}$, generally below $0.20 \mathrm{apfu}$ in primary Tur IB, increases up to $0.755 \mathrm{Zn}$ apfu (6.32 wt \% ZnO) in Tur IIIA and up to $0.880 \mathrm{Zn}$ apfu (7.37 wt \% $\mathrm{ZnO}$ ) in Tur IIIB. Thus, in the absence of $\mathrm{Mg}$, the content of bivalent cations $\mathrm{Zn}+\mathrm{Fe}^{2+}+\mathrm{Mn}^{2+}$ varies from 0.990 to 1.175 apfu in Tur IIIA and from 0.933 to 1.148 apfu in Tur IIIB, with $\mathrm{Zn}>>\mathrm{Fe}^{2+}>\mathrm{Mn}^{2+}$. However, because in all acquired compositions $\mathrm{Zn}+\mathrm{Fe}^{2+}+\mathrm{Mn}^{2+}<1.5$ apfu, they do not correspond to $\mathrm{Zn}$-dominant tourmaline of the hypothetical formula $\mathrm{NaZn}_{3} \mathrm{Al}_{6} \mathrm{~B}_{3} \mathrm{Si}_{6} \mathrm{O}_{27}(\mathrm{OH})_{3}(\mathrm{OH})$ (Figure 6). This hypothetical end-member reaches the maximum content of about $40 \mathrm{~mol} \%$, and $\mathrm{Zn}$ can only be the most important second-rank component in the tourmalines. The most $\mathrm{Zn}$-enriched compositions yield the formulae: $\mathrm{X}\left(\mathrm{Na}_{0.733} \mathrm{Ca}_{0.013} \square_{0.254}\right)_{\Sigma 1}{ }^{\mathrm{Y}}\left(\mathrm{Al}_{1.033} \mathrm{Li}_{0.792} \mathrm{Zn}_{0.755} \mathrm{Fe}^{2+}{ }_{0.326} \mathrm{Mn}_{0.094}\right)_{\Sigma 3}{ }^{\mathrm{Z}} \mathrm{Al}_{6}\left({ }^{\mathrm{T}} \mathrm{Si}_{6} \mathrm{O}_{18}\right)\left(\mathrm{BO}_{3}\right)_{3}{ }^{\mathrm{V}}(\mathrm{OH})_{3}{ }^{\mathrm{W}}\left(\mathrm{F}_{0.654}\right.$ $\left.\mathrm{OH}_{0.344}\right)$ for Tur IIIA and ${ }^{\mathrm{X}}\left(\mathrm{Na}_{0.779} \mathrm{Ca}_{0.015} \square_{0.206}\right)_{\Sigma 1}{ }^{\mathrm{Y}}\left(\mathrm{Al}_{1.061} \mathrm{Li}_{0.869} \mathrm{Zn}_{0.880} \mathrm{Fe}^{2+}{ }_{0.098} \mathrm{Mn}_{0.094}\right)_{\Sigma 3}{ }^{\mathrm{Z}} \mathrm{Al}_{6}\left({ }^{\mathrm{T}} \mathrm{Si}_{6} \mathrm{O}_{18}\right)$ $\left(\mathrm{BO}_{3}\right)_{3}{ }^{\mathrm{V}}(\mathrm{OH})_{3}{ }^{\mathrm{W}}\left(\mathrm{OH}_{0.837} \mathrm{~F}_{0.163}\right)$ for Tur IIIB.

The extent of Mn-Fe fractionation increases from Tur IIIA to Tur IIIB, with $\mathrm{Mn} /(\mathrm{Mn}+\mathrm{Fe})$ ranging from 0.166 to 0.286 and from 0.293 to 0.517 , respectively. However, because $\mathrm{Zn}$ fractionates along with $\mathrm{Mn}$, the ratio $(\mathrm{Mn}+\mathrm{Zn}) /(\mathrm{Mn}+\mathrm{Zn}+\mathrm{Fe}+\mathrm{Mg})$ is a better fractionation index yielding, respectively, $0.490-0.759$ and $0.827-0.938$. 


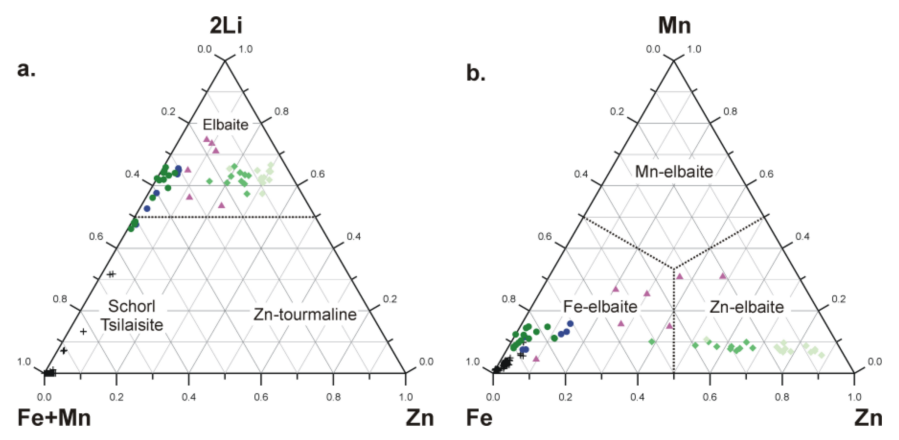

Figure 6. Compositional plots of tourmalines from the pegmatite that contain $\mathrm{Zn}$-rich fluor-elbaite and Zn-rich elbaite in the system: (a) schorl+tsilaisite—elbaite-hypothetical Zn-tourmaline; (b) Fe-Mn-Zn, showing relationships between second-rank divalent Y-site occupants. Symbols: dark crosses-primary schorl-type tourmaline Tur I, dark blue circles-inclusions of dark blue elbaite and fluor-elbaite (Tur IIA), dark green circles-green fluor-elbaite from mica books (Tur IIB), green diamonds-Zn-rich fluor-elbaite (Tur IIIA), pale green diamonds-Zn-rich elbaite (Tur IIIB), violet triangles-Zn-bearing tourmalines from the mineralogical literature [10-14,16].

\subsection{Compositional Relationships}

Figure 7 presents variations in concentrations of the most important elements in the tourmalines as functions of $\mathrm{Mn}+\mathrm{Zn}$ fractionation expressed by the ratio $(\mathrm{Mn}+\mathrm{Zn}) /(\mathrm{Mn}+\mathrm{Zn}+\mathrm{Mg}+\mathrm{Fe})$. Mafic elements, such as $\mathrm{Mg}, \mathrm{Fe}^{2+}$ and Ti display rather coherent decreasing trends (Figure $7 \mathrm{a}-\mathrm{c}$ ). While present as minor components in Tur IA, Mg and Ti disappear in Tur IB, and may be present only as traces in more fractionated Tur II and Tur III. Iron decreases rapidly from Tur IA to Tur IIB, reaching a relatively constant content of $\sim 0.70-0.75 \mathrm{apfu}$. Tur III shows a further steady decrease down to $0.058 \mathrm{Fe}$ apfu along with $\mathrm{Mn}+\mathrm{Zn}$ fractionation. Calcium, a subordinate component in Ti-bearing Tur IA, decreases very rapidly to a trace level in Tur IB (Figure 7d). However, there is a weak increase in Tur IIA-IIB at the $(\mathrm{Mn}+\mathrm{Zn}) /(\mathrm{Mn}+\mathrm{Zn}+\mathrm{Mg}+\mathrm{Fe})$ of $0.30-0.40$, followed by a regular decrease in more fractionated compositions. Zinc, $\mathrm{Mn},{ }^{\mathrm{Y}} \mathrm{Al}, \mathrm{Na}$ and $\mathrm{F}$ show, at least partly, opposite tendencies (Figure 7e-i). There is a sharp increase of $\mathrm{Mn}$ and $\mathrm{Al}$ contents in Tur I owing to Mn-Fe fractionation, as well as dehydroxylation and alkali-vacant substitutions: $\mathrm{Al}^{3+}+\mathrm{O}^{2-} \rightarrow{ }^{\mathrm{Y}}(\mathrm{Fe}, \mathrm{Mg})^{2+}+\mathrm{OH}^{-}$and $\mathrm{Al}^{3+}+\square \rightarrow{ }^{\mathrm{Y}}(\mathrm{Fe}, \mathrm{Mg})^{2+}+{ }^{\mathrm{X}} \mathrm{Na}^{+}$, respectively. In Zn-poor Tur IIA and Tur IIB, both components fluctuate around values of 0.10-0.15 Mn apfu and $~ 1.0-1.3 \mathrm{Al} a p f u$. The appearance of $\mathrm{Li}$ in tourmaline (from Tur IB to Tur III) changes the compositional relationships between ${ }^{\mathrm{Y}} \mathrm{Al}$ and divalent $\mathrm{Y}$-site cations due to the coupled substitution $\mathrm{Li}^{+}+\mathrm{Al}^{3+}+\mathrm{F}^{-} \rightarrow 2{ }^{\mathrm{Y}} \mathrm{Fe}^{2+}+{ }^{\mathrm{W}} \mathrm{OH}^{-}$at the $\mathrm{Y}$ and $\mathrm{W}$ sites. This leads to the crystallization of fluor-elbaite and elbaite (some compositions of Tur II). As a result, $\mathrm{Al}, \mathrm{Li}$ and $\mathrm{Fe}^{2+}+\mathrm{Mn}^{2+}+\mathrm{Zn}$ become the only significant $\mathrm{Y}$-site occupants in more fractionated tourmalines. Further compositional evolution is a result of local fluctuation among activities of these components, especially due to dissolution and reprecipitation caused by the (Na,Li,F,B)-enriched fluid, and $\mathrm{Mn}+\mathrm{Zn}$ vs. Fe fractionation.

With fractionation, zinc forms a well-defined increasing trend (Figure 7e), reaching $0.020 \mathrm{apfu}$ (0.16 wt \% ZnO) in Tur IA, 0.061 apfu (0.48 wt \% ZnO) in Tur IB, 0.141 apfu (1.17 wt \% ZnO) in Tur IIA, and 0.111 apfu $(0.93 \mathrm{wt} \% \mathrm{ZnO})$ in Tur IIB. Dissolution of gahnite and primary Tur IB and crystallization of secondary Zn-rich Tur III are marked by a sharp increase of Zn content up to $6.32 \mathrm{wt} \% \mathrm{ZnO}$ (0.755 Zn apfu) in Tur IIIA and to $7.37 \mathrm{wt} \%(0.880 \mathrm{Zn} a p f u)$ in Tur IIIB. The stage of atypically high enrichment in $\mathrm{Zn}$ in these tourmalines is clearly shown in the $(\mathrm{Mn}+\mathrm{Zn}) /(\mathrm{Mn}+\mathrm{Zn}+\mathrm{Mg}+\mathrm{Fe}) \mathrm{vs}$. $\mathrm{Mn} /(\mathrm{Mn}+\mathrm{Fe})$ plot (Figure 7j). These two parameters show a simple linear relationship in all tourmaline generations, in which the increasing content of $\mathrm{Zn}$ can be attributed only to geochemical fractionation. In the relatively less evolved tourmalines (Tur I-IIB), the maximum values of the two ratios are relatively low, less than 0.20 and 0.30 for $\mathrm{Mn} /(\mathrm{Mn}+\mathrm{Fe})$ and $(\mathrm{Mn}+\mathrm{Zn}) /(\mathrm{Mn}+\mathrm{Zn}+\mathrm{Mg}+\mathrm{Fe})$, respectively. In Tur III adjacent to 
gahnite, Zn suddenly increases, which is marked by the interruption of the trend and a sudden jump of the $(\mathrm{Mn}+\mathrm{Zn}) /(\mathrm{Mn}+\mathrm{Zn}+\mathrm{Mg}+\mathrm{Fe})$ value to $\sim 0.5-0.7$. However, the trend is back to normal at the stage of Tur IIIB crystallization, which suggests the dominance of Mn and Zn fractionation over dissolution-reprecipitation. Such behavior is synchronous with a distinct decrease in F activity, reflected in a gradual transition from the crystallization of fluor-elbaite (Tur IIIA) to elbaite (Tur IIIB) (Figure 7i).
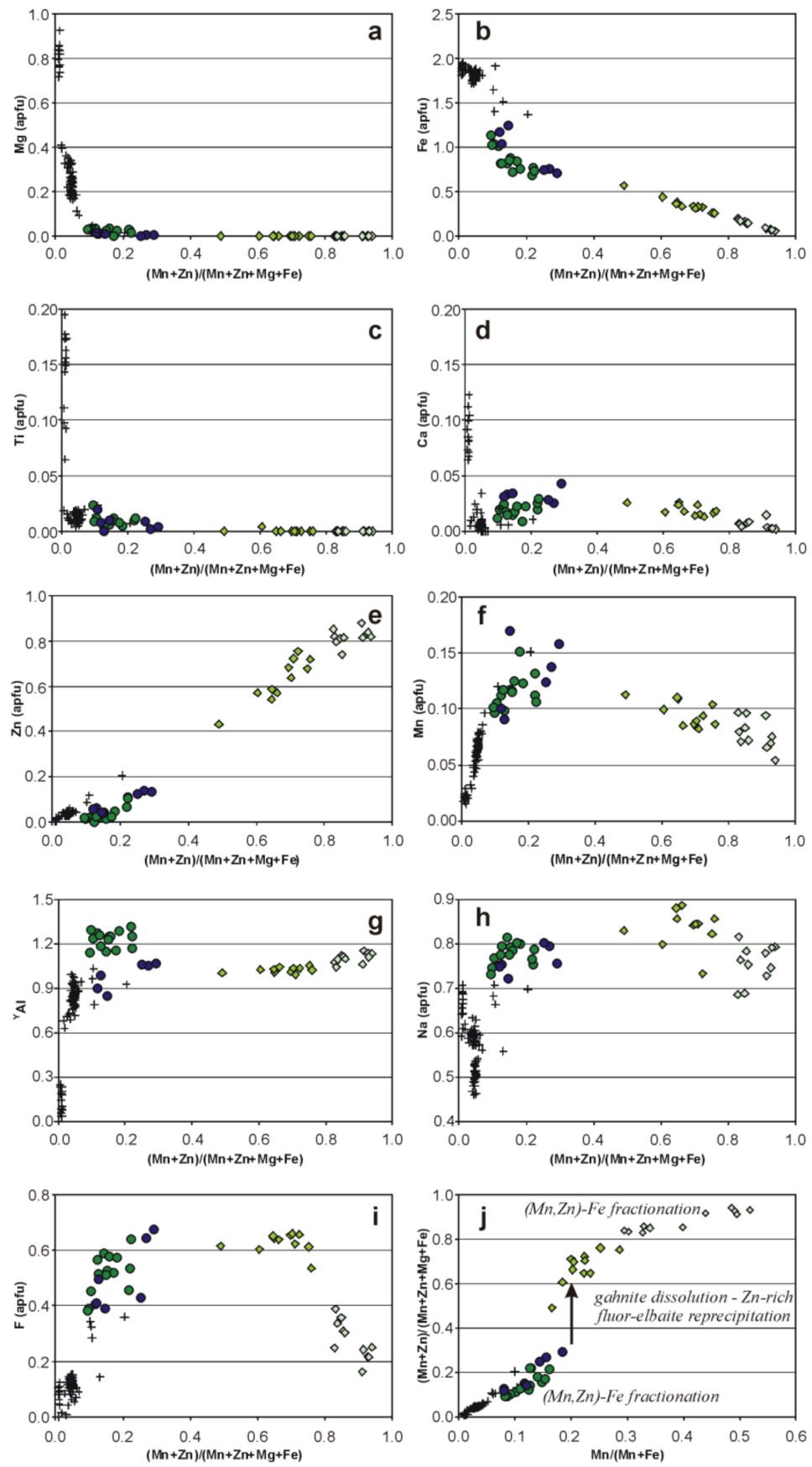

Figure 7. Compositional relationships in tourmalines evolving to $\mathrm{Zn}$-rich fluor-elbaite and elbaite: (a) $\mathrm{Mg}$ vs. $(\mathrm{Mn}+\mathrm{Zn}) /(\mathrm{Mn}+\mathrm{Zn}+\mathrm{Mg}+\mathrm{Fe}) ;(\mathbf{b}) \mathrm{Fe}$ vs. $(\mathrm{Mn}+\mathrm{Zn}) /(\mathrm{Mn}+\mathrm{Zn}+\mathrm{Mg}+\mathrm{Fe}) ;(\mathbf{c}) \mathrm{Ti}$ vs. $(\mathrm{Mn}+$ $\mathrm{Zn}) /(\mathrm{Mn}+\mathrm{Zn}+\mathrm{Mg}+\mathrm{Fe}) ;(\mathbf{d})$ Ca vs. $(\mathrm{Mn}+\mathrm{Zn}) /(\mathrm{Mn}+\mathrm{Zn}+\mathrm{Mg}+\mathrm{Fe}) ;(\mathbf{e}) \mathrm{Zn}$ vs. $(\mathrm{Mn}+\mathrm{Zn}) /(\mathrm{Mn}+$ $\mathrm{Zn}+\mathrm{Mg}+\mathrm{Fe}) ;(\mathbf{f}) \mathrm{Mn}$ vs. $(\mathrm{Mn}+\mathrm{Zn}) /(\mathrm{Mn}+\mathrm{Zn}+\mathrm{Mg}+\mathrm{Fe}) ;(\mathrm{g}) \mathrm{YAl}$ vs. $(\mathrm{Mn}+\mathrm{Zn}) /(\mathrm{Mn}+\mathrm{Zn}+\mathrm{Mg}+$ $\mathrm{Fe}) ;(\mathbf{h}) \mathrm{Na}$ vs. $(\mathrm{Mn}+\mathrm{Zn}) /(\mathrm{Mn}+\mathrm{Zn}+\mathrm{Mg}+\mathrm{Fe}) ;(\mathbf{i}) \mathrm{F}$ vs. $(\mathrm{Mn}+\mathrm{Zn}) /(\mathrm{Mn}+\mathrm{Zn}+\mathrm{Mg}+\mathrm{Fe})$, (j) $(\mathrm{Mn}+$ $\mathrm{Zn}) /(\mathrm{Mn}+\mathrm{Zn}+\mathrm{Mg}+\mathrm{Fe})$ vs. Mn/(Mn $+\mathrm{Fe})$. Symbols as in Figure 6. 


\subsection{Raman Spectroscopy}

Raman spectroscopy is a widely applied method for the identification of minerals as well as recognition of their compositional relationships; it is also used frequently in studies of tourmalines [36-44]. Because of the particular location of $\mathrm{OH}$ groups, which are bound to the octahedral $\mathrm{Y}$ and $\mathrm{Z}$ cations in the tourmaline structure, the $\mathrm{OH}$ vibration bands code crystallochemical information on occupation of both octahedral sites. Therefore, interpretation of the bands is useful in discussing distribution of octahedral cations between the $\mathrm{Y}$ and $\mathrm{Z}$ sites (e.g., [39,40,42-45]).

Figure 8 shows representative unoriented Raman spectra recorded on relicts of primary Li,Al-bearing schorl Tur IB and secondary fluor-elbaite Tur IIIA (with compositions close to analyses 225/2 and 225/4 presented in Table 3). Generally, the spectra resemble those for schorl and fluor-elbaite presented by Watenphul et al. ([44]; Figure 3), with bands in the wavenumber range of 50-1200 $\mathrm{cm}^{-1}$ interpreted as results from $\mathrm{Me}-\mathrm{O}$ stretching vibrations on the $\mathrm{Y}$ and $\mathrm{Z}$ sites, $\mathrm{Si}-\mathrm{O}$ vibrations in the $\mathrm{Si}_{6} \mathrm{O}_{18}$ ring, and stretching vibrations of the $\mathrm{B}-\mathrm{O}$ bonds [37-45].

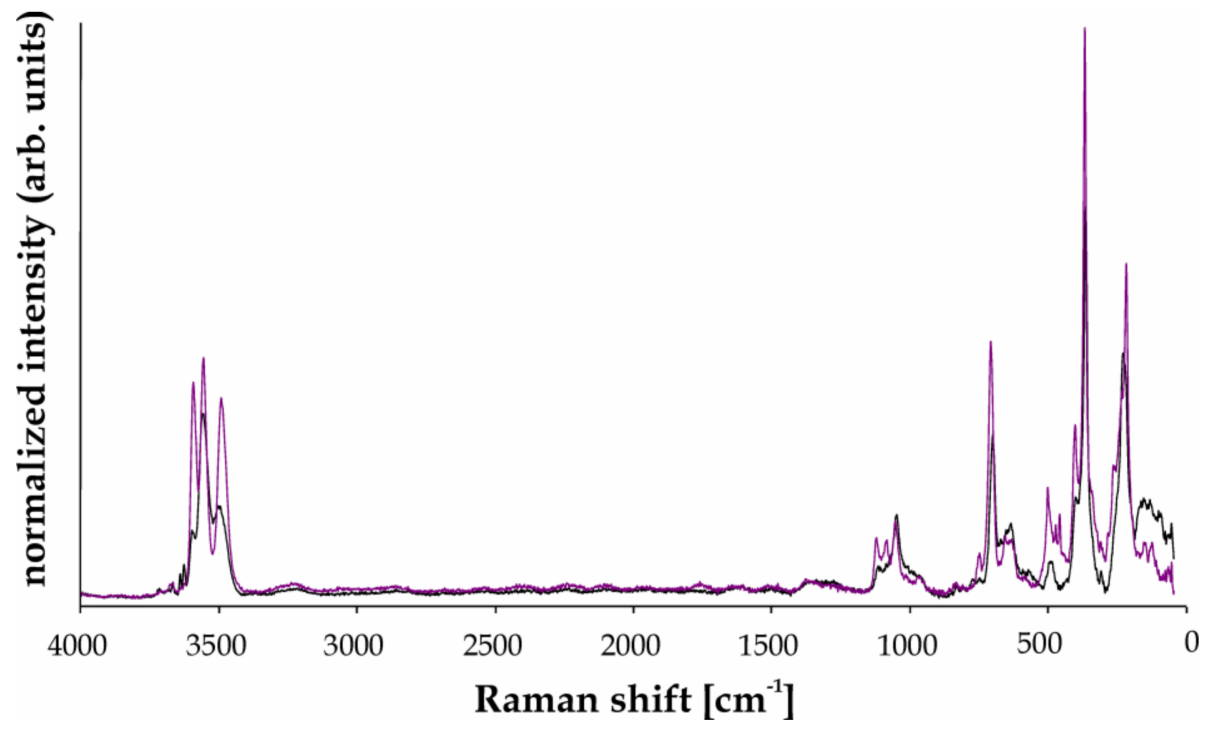

Figure 8. Raman spectra of schorl-type tourmaline Tur IB (black) and Zn-rich fluor-elbaite Tur IIIA (violet) in the range $50-4000 \mathrm{~cm}^{-1}$.

In the range of $\mathrm{OH}$ stretching vibration modes $\left(3350-3800 \mathrm{~cm}^{-1}\right)$, the spectrum of Zn-rich Tur IIIA has three intense peaks with maxima at 3497, 3561 and $3597 \mathrm{~cm}^{-1}$ (Figure 9a). Peaks with similar Raman shifts in the spectrum of fluor-elbaite $\left(3494 \pm 8,3562 \pm 4\right.$ and $\left.3593 \pm 4 \mathrm{~cm}^{-1}\right)$ were related by Watenphul et al. [44] to the most probable variants of configurations of the octahedral cations in this tourmaline variety: ${ }^{\mathrm{Y}} \mathrm{Li}^{\mathrm{Z}} \mathrm{Al}^{\mathrm{Z}} \mathrm{Al}-2^{\mathrm{Y}} \mathrm{Al}^{\mathrm{Z}} \mathrm{Al}^{\mathrm{Z}} \mathrm{Al}, 2^{\mathrm{Y}} \mathrm{Fe}^{* 2+\mathrm{Z}} \mathrm{Al}^{\mathrm{Z}} \mathrm{Al}-{ }^{\mathrm{Y}} \mathrm{Al}^{\mathrm{Z}} \mathrm{Al}^{\mathrm{Z}} \mathrm{Al}$ and $2^{\mathrm{Y}} \mathrm{Li}^{\mathrm{Z}} \mathrm{Al}^{\mathrm{Z}} \mathrm{Al}^{\mathrm{Y}} \mathrm{Al}^{\mathrm{Z}} \mathrm{Al}^{\mathrm{Z}} \mathrm{Al}$, where ${ }^{\mathrm{Y}} \mathrm{Fe}^{* 2+}$ denotes the total of all divalent $\mathrm{Y}$-site occupants $\left({ }^{\mathrm{Y}} \mathrm{Fe}^{* 2+}={ }^{\mathrm{Y}} \mathrm{Fe}^{2+}+{ }^{\mathrm{Y}} \mathrm{Mn}^{2+}+{ }^{\mathrm{Y}} \mathrm{Zn}^{2+}+\right.$ ${ }^{\mathrm{Y}} \mathrm{Mg}^{2+}$ ). A visible small asymmetry of the peak $3497 \mathrm{~cm}^{-1}$ centered at $\sim 3475 \mathrm{~cm}^{-1}$ could come from ${ }^{\mathrm{Y}} \mathrm{Fe}^{* 2+\mathrm{Z}} \mathrm{Al}^{\mathrm{Z}} \mathrm{Al}-2^{\mathrm{Y}} \mathrm{Al}^{\mathrm{Z}} \mathrm{Al}^{\mathrm{Z}} \mathrm{Al}$ complexes of $\mathrm{Y}$ and $\mathrm{Z}$ octahedra in the interpretation of Watenphul et al. [44].

However, we would like to discuss the positions and intensities of the observed bands adopting a slightly different approach (Table 4). Because in the tourmaline structure, ${ }^{\mathrm{V}} \mathrm{OH}$ groups occur with an at least $75 \%$ abundance relative to the total content of hydroxyls [3VOH $+\mathrm{W}(\mathrm{OH}, \mathrm{F}, \mathrm{O})]$, the resulting Raman bands have relatively high intensities. The ${ }^{\mathrm{V}} \mathrm{OH}$ groups are bound to one $\mathrm{Y}$ and two $\mathrm{Z}$ atoms in the tourmaline structure. Therefore, in all Li-bearing tourmalines, with the $\mathrm{Z}$ site occupied exclusively by $\mathrm{Al}$, only ${ }^{\mathrm{Y}} \mathrm{Al}^{\mathrm{Z}} \mathrm{Al}^{\mathrm{Z}} \mathrm{Al}$, ${ }^{\mathrm{Y}} \mathrm{Fe}^{* 2+\mathrm{Z}} \mathrm{Al}^{\mathrm{Z}} \mathrm{Al}$ and ${ }^{\mathrm{Y}} \mathrm{Li}^{\mathrm{Z}} \mathrm{Al}^{\mathrm{Z}} \mathrm{Al}$ arrangements are possible for the $\mathrm{YZZ}$ triad around the $\mathrm{V}=\mathrm{O}(3)$ site. The ${ }^{\mathrm{Z}} \mathrm{Al}-\mathrm{O}(3)$ and ${ }^{\mathrm{Y}} \mathrm{Al}-\mathrm{O}(3)$ are the longest bonds in the $\mathrm{Z}$ and $\mathrm{Y}$ octahedra, e.g., [46], but the differences with other $\mathrm{Z}-\mathrm{O}$ or $\mathrm{Y}-\mathrm{O}$ bonds seems rather too small among various tourmaline species to explain such significant differences in Raman shift of 
the resulting bands. Similarly, this spectral differentiation cannot be explained by different atomic masses of the $\mathrm{Y}$ occupants $(\mathrm{Li}=6.94, \mathrm{Al}=26.98, \mathrm{Fe}=55.85, \mathrm{Zn}=65.38, \ldots)$, because the positions of the absorption bands assigned to different variants of configurations of octahedral cations do not correlate with these data. Therefore, the only plausible explanation seems to be that when distribution of electrons around their nuclei is not-fully spherical, different positive charges of the Y-occupants $(3+, 2+$ or $1+)$ draw the electron density away from the ${ }^{\mathrm{V}} \mathrm{O}-\mathrm{H}$ bond. As a result, the bond length increases, but its strength decreases, in accordance with the sequence ${ }^{\mathrm{Y}} \mathrm{Al}^{3+} \ldots{ }^{\mathrm{V}} \mathrm{O}-\mathrm{H}$ (the largest $\mathrm{O}-\mathrm{H}$ distance; the lowest Raman shift), ${ }^{\mathrm{Y}} \mathrm{Fe}^{* 2+} \ldots{ }^{\mathrm{V}} \mathrm{O}-\mathrm{H}$, and ${ }^{\mathrm{Y}} \mathrm{Li}^{+} \ldots{ }^{\mathrm{V}} \mathrm{O}-\mathrm{H}$ (the shortest $\mathrm{O}-\mathrm{H}$ distance; the highest Raman shift). This interpretation model is consistent with some previous explanations of $\mathrm{OH}$ stretching vibration modes in various tourmaline varieties (e.g., $[40,42,45])$. The peak around $3497 \mathrm{~cm}^{-1}$ with visible small asymmetry in the spectrum of Zn-rich fluor-elbaite Tur IIIA, related generally to ${ }^{\mathrm{V} O H}-{ }^{\mathrm{Y}} \mathrm{Al}^{\mathrm{Z}} \mathrm{Al}^{\mathrm{Z}} \mathrm{Al}$, we interpret as superposition of two bands corresponding to ${ }^{\mathrm{V}} \mathrm{OH}$ vibrations modified by the ${ }^{\mathrm{Y}} \mathrm{Al}^{\mathrm{Z}} \mathrm{Al}^{\mathrm{Z}} \mathrm{Al}$ arrangement associated with the Na-occupied or vacant $\mathrm{X}$ site.

The spectrum of Li,Al-bearing schorl-type tourmaline Tur IB (Figure 9b), with three visible maxima in the range of Raman shift below $3600 \mathrm{~cm}^{-1}$, is dominated by $3562 \mathrm{~cm}^{-1}$ absorption, which is interpreted as dominantly coming from stretching vibrations in the ${ }^{\mathrm{V}_{\mathrm{OH}}}{ }^{\mathrm{Y}} \mathrm{Fe}^{* 2+\mathrm{Z}} \mathrm{Al}^{\mathrm{Z}} \mathrm{Al}$ arrangement. The two remaining maxima are related to ${ }^{\mathrm{V}} \mathrm{OH}_{-}{ }^{\mathrm{Y}} \mathrm{Li}^{\mathrm{Z}} \mathrm{Al}^{\mathrm{Z}} \mathrm{Al}\left(3602 \mathrm{~cm}^{-1}\right)$ and a relatively wide unresolved band $\left(3503 \mathrm{~cm}^{-1}\right)$, which we interpret as a superposition of ${ }^{\mathrm{V}} \mathrm{OH}_{-}{ }^{\mathrm{Y}} \mathrm{Al}^{\mathrm{Z}} \mathrm{Al}^{\mathrm{Z}} \mathrm{Al}_{-}{ }^{\mathrm{X}} \mathrm{Na}$ and ${ }^{\mathrm{V}} \mathrm{OH}-{ }^{\mathrm{Y}} \mathrm{Al}^{\mathrm{Z}} \mathrm{Al}^{\mathrm{Z}} \mathrm{Al}-{ }^{\mathrm{X}} \square$ bands, similarly as in the case of Tur IIIA.

Table 4. Assignments of the O-H stretching bands in the Raman spectra of Tur IB (fluor-schorl) and Tur IIIA (Zn-rich fluor-elbaite).

\begin{tabular}{|c|c|c|c|c|c|c|c|}
\hline \multicolumn{4}{|c|}{ Tur IB } & \multicolumn{4}{|c|}{ Tur IIIA } \\
\hline \multicolumn{4}{|c|}{$\mathrm{Fe}^{* 2+}: \mathrm{Zn}<<\mathrm{Fe}+\mathrm{Mn}$} & \multicolumn{4}{|c|}{$\mathrm{Fe}^{* 2+}: \mathrm{Zn}>>\mathrm{Fe}+\mathrm{Mn}$} \\
\hline $\begin{array}{c}\text { Band } \\
\left(\mathrm{cm}^{-1}\right)\end{array}$ & $\begin{array}{l}\text { Rel. int. } \\
(\%)\end{array}$ & $\begin{array}{c}\text { Calc. } \\
\text { int. }(\%)\end{array}$ & Assignment & $\begin{array}{c}\text { Band } \\
\left(\mathrm{cm}^{-1}\right)\end{array}$ & $\begin{array}{l}\text { Rel. int. } \\
(\%)\end{array}$ & $\begin{array}{c}\text { Calc. } \\
\text { int. (\%) }\end{array}$ & Assignment \\
\hline \multirow{3}{*}{3503} & \multirow{3}{*}{41} & \multirow{3}{*}{28} & \multirow{3}{*}{${ }^{\mathrm{V}} \mathrm{OH}_{-}{ }^{\mathrm{Y}} \mathrm{Al}^{+\mathrm{Z}} \mathrm{Al}^{\mathrm{Z}} \mathrm{Al}$} & 3478 & 17 & \multirow[b]{2}{*}{31} & ${ }^{\mathrm{V}} \mathrm{OH}_{-}{ }^{\mathrm{Y}} \mathrm{Al}^{\mathrm{Z}} \mathrm{Al}^{\mathrm{Z}} \mathrm{Al}-{ }^{\mathrm{X}} \square$ \\
\hline & & & & 3497 & 18 & & ${ }^{\mathrm{V}} \mathrm{OH}-{ }^{\mathrm{Y}} \mathrm{Al}^{\mathrm{Z}} \mathrm{Al}^{\mathrm{Z}} \mathrm{Al}-{ }^{\mathrm{X}} \mathrm{Na}$ \\
\hline & & & & 3556 & 11 & (9) & ${ }^{\mathrm{w}} \mathrm{OH}-\ldots \ldots{ }_{-}^{\mathrm{x}} \mathrm{Na}$ \\
\hline 3562 & 43 & 53 & ${ }^{\mathrm{V}} \mathrm{OH}-{ }^{\mathrm{Y}} \mathrm{Fe}^{* 2+\mathrm{Z}} \mathrm{Al}^{\mathrm{Z}} \mathrm{Al}$ & 3561 & 34 & 31 & ${ }^{\mathrm{V}} \mathrm{OH}-{ }^{\mathrm{Y}} \mathrm{Fe}^{* 2+\mathrm{Z}} \mathrm{Al}^{\mathrm{Z}} \mathrm{Al}$ \\
\hline 3602 & 8 & 10 & ${ }^{\mathrm{V}} \mathrm{OH}_{-}{ }^{\mathrm{Y}} \mathrm{Li}^{\mathrm{Z}} \mathrm{Al}^{\mathrm{Z}} \mathrm{Al}$ & 3597 & 18 & 27 & ${ }^{\mathrm{V}} \mathrm{OH}_{-}{ }^{\mathrm{Y}} \mathrm{Li}^{\mathrm{Z}} \mathrm{Al}^{\mathrm{Z}} \mathrm{Al}$ \\
\hline 3630 & 3 & 2 & ${ }^{\mathrm{W}} \mathrm{OH}_{-}{ }^{\mathrm{Y}} \mathrm{Fe}^{* 2+\mathrm{Y}} \mathrm{Al}^{\mathrm{Y}} \mathrm{Al}^{-}{ }^{\mathrm{X}} \square$ & - & - & - & \\
\hline 3644 & 1 & 1 & ${ }^{\mathrm{W}} \mathrm{OH}-{ }^{\mathrm{Y}} \mathrm{Fe}^{* 2+\mathrm{Y}} \mathrm{Fe}^{* 2+\mathrm{Y}} \mathrm{Al}-{ }^{\mathrm{X}} \square$ & - & - & - & ${ }^{\mathrm{W}} \mathrm{OH}-{ }^{\mathrm{Y}} \mathrm{Fe}^{* 2+\mathrm{Y}} \mathrm{Fe}^{* 2+\mathrm{Y}} \mathrm{Al}-{ }^{\mathrm{X}} \square$ \\
\hline 3671 & 1 & 1 & ${ }^{\mathrm{W}} \mathrm{OH}-{ }^{\mathrm{Y}} \mathrm{Li}^{\mathrm{Y}} \mathrm{Fe}^{* 2+\mathrm{Y}} \mathrm{Al}-{ }^{\mathrm{X}} \square$ & 3676 & 1 & 1 & ${ }^{\mathrm{W}} \mathrm{OH}-{ }^{\mathrm{Y}} \mathrm{Li}^{\mathrm{Y}} \mathrm{Fe}^{* 2+\mathrm{Y}} \mathrm{Al}-{ }^{\mathrm{X}} \square$ \\
\hline 3688 & 2 & 2 & ${ }^{\mathrm{W}} \mathrm{OH}-{ }^{\mathrm{Y}} \mathrm{Fe}^{* 2+\mathrm{Y}} \mathrm{Fe}^{* 2+\mathrm{Y}} \mathrm{Al}-{ }^{\mathrm{X}} \mathrm{Na}$ & - & - & - & ${ }^{\mathrm{W}} \mathrm{OH}-{ }^{\mathrm{Y}} \mathrm{Fe}^{* 2+Y} \mathrm{Fe}^{* 2+\mathrm{Y}} \mathrm{Al}-{ }^{X} \mathrm{Na}$ \\
\hline 3720 & 1 & 2 & ${ }^{W} \mathrm{OH}-{ }^{Y} \mathrm{Fe}^{* 2+Y} \mathrm{Fe}^{* 2+Y} \mathrm{Fe}^{* 2+}{ }^{X} \mathrm{Na}$ & 3709 & 1 & 1 & ${ }^{\mathrm{W}} \mathrm{OH}-{ }^{\mathrm{Y}} \mathrm{Fe}^{* 2+\mathrm{Y}} \mathrm{Fe}^{* 2+\mathrm{Y}} \mathrm{Fe}^{* 2+}{ }^{\mathrm{X}} \mathrm{Na}$ \\
\hline
\end{tabular}

The ${ }^{\mathrm{W}} \mathrm{OH}$ group is bound to three $\mathrm{Y}$ occupants. The presence of $\mathrm{Al}, \mathrm{Fe}^{* 2+}$ and $\mathrm{Li}$ at the $\mathrm{Y}$ site of the described Zn-rich fluor-elbaite results in eight allowed models of the YYY triad (LiLiLi and $\mathrm{LiLiFe}^{* 2+}$ local arrangements are not allowed because local anion bond valence requirements around the $\mathrm{W}=\mathrm{O}(1)$ site would not be satisfied [47]). It should be expected that with the small proportion of ${ }^{\mathrm{W}} \mathrm{OH}$ in the total $\mathrm{OH}$ content $(<25 \%)$ the abundances of individual YYY arrangements should be subordinate and the resulting intensities of the respective stretching vibration bands should be low. Therefore, they only have the potential to modify the basic pattern of the spectrum configured essentially by ${ }^{\mathrm{V}} \mathrm{OH}$ bands. Applying to the YYY cationic arrangements the same reasoning as the one used above for the YZZ triad, it can be observed that the strongest effect of shifting the electron density away from the ${ }^{\mathrm{W}} \mathrm{O}-\mathrm{H}$ bond would take place in the ${ }^{\mathrm{Y}} \mathrm{Al}^{\mathrm{Y}} \mathrm{Al}^{\mathrm{Y}} \mathrm{Al}$ arrangement, and the weakest for the ${ }^{\mathrm{Y}} \mathrm{Li}^{\mathrm{Y}} \mathrm{Li}^{\mathrm{Y}} \mathrm{Al}$ triad. Consequently, the ${ }^{\mathrm{W}} \mathrm{O}-\mathrm{H}$ bond should be the weakest in case of the first-type triad, and the strongest for the last. 

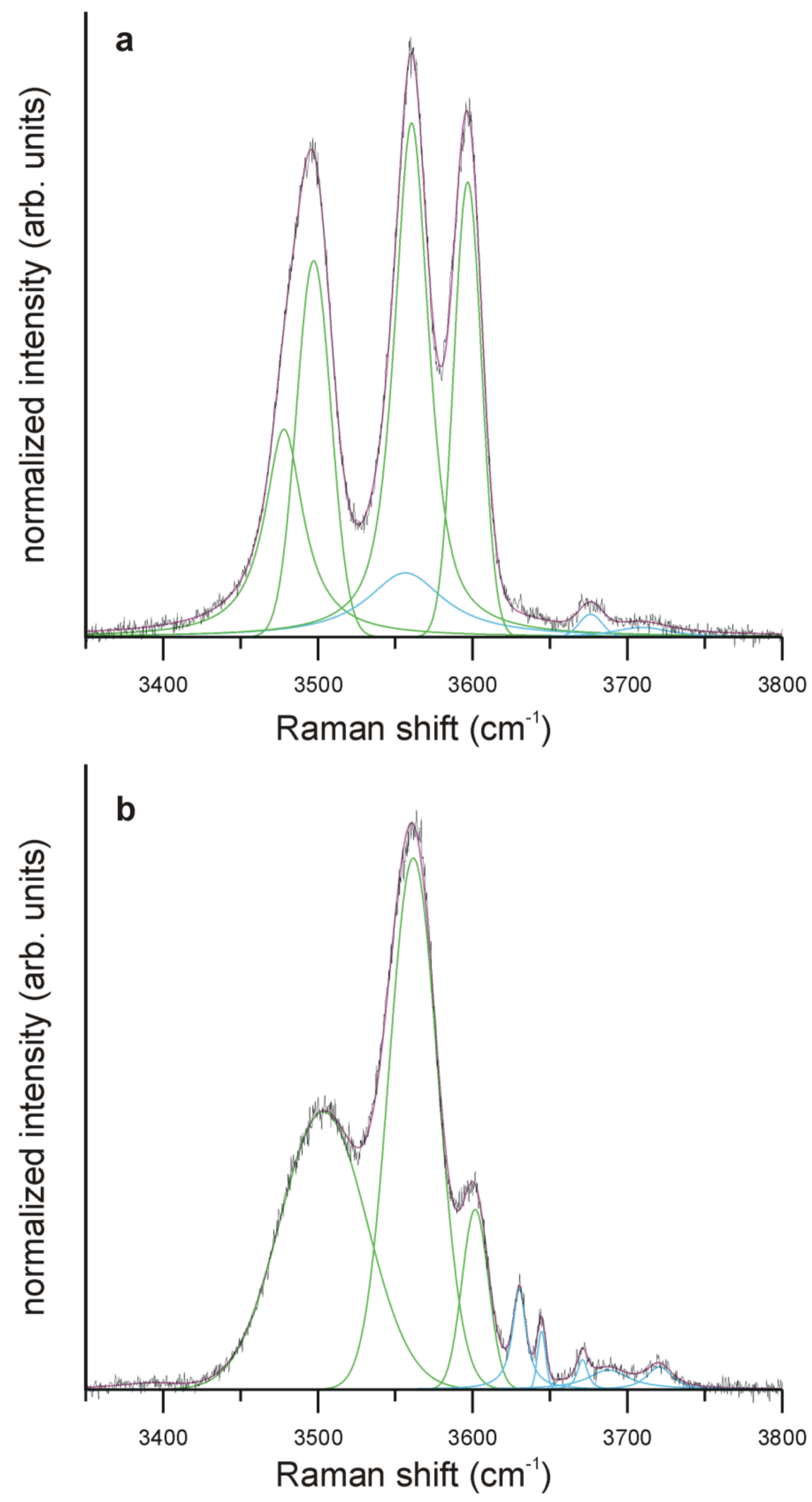

Figure 9. Representative Raman spectra of: (a) primary schorl-type tourmaline (Tur IB) and (b) Zn-rich fluor-elbaite (Tur IIIA) in the range of $\mathrm{OH}$ stretching vibrations. Line colors: black-the recorded spectrum, red-the fitted spectrum; green-bands dominated by ${ }^{\mathrm{V}} \mathrm{OH}$ stretching vibrations, blue-bands dominated by ${ }^{\mathrm{W}} \mathrm{OH}$ stretching vibrations. 
In the case of absence of $\mathrm{Na}$ or $\mathrm{Ca}$ at the $\mathrm{X}$ site (i.e., the presence of $\mathrm{X}$-site vacancy as e.g., in foitite), Watenphul et al. ([44]; Figure 5) observed a few subordinate bands at wavenumbers generally above $3600 \mathrm{~cm}^{-1}$. They assigned them to stretching vibrations in ${ }^{\mathrm{W}} \mathrm{OH}$ groups bound to a YYY triad and associated with an unoccupied $X$ site $(Y Y Y-X \square)$ or the site occupied by Na (YYY-X Na). The X-site vacancy is a result of a common substitution in the tourmaline structure: $\square+\mathrm{Me}^{3+} \rightarrow{ }^{\mathrm{X}} \mathrm{Na}++{ }^{\mathrm{Y}} \mathrm{Me}^{2+}$. In this substitution, $\mathrm{Me}^{3+}$ cation replaces $\mathrm{Me}^{2+}$ in one octahedron of the $\mathrm{Y}$ triad. This means that the only valid YYY cationic arrangements in the structure of various tourmaline species with vacancy at the $\mathrm{X}$ site are: ${ }^{\mathrm{Y}} \mathrm{Al}^{\mathrm{Y}} \mathrm{Al}^{\mathrm{Y}} \mathrm{Al}-{ }^{\mathrm{X}} \square,{ }^{\mathrm{Y}} \mathrm{Fe}^{* 2+\mathrm{Y}} \mathrm{Al}^{\mathrm{Y}} \mathrm{Al}-{ }^{\mathrm{X}} \square,{ }^{\mathrm{Y}} \mathrm{Fe}^{* 2+\mathrm{Y}} \mathrm{Fe}^{* 2+\mathrm{Y}} \mathrm{Al}_{-}{ }^{\mathrm{X}} \square,{ }^{\mathrm{Y}} \mathrm{Li}^{\mathrm{Y}} \mathrm{Fe}^{* 2+\mathrm{Y}} \mathrm{Al}^{-}{ }^{\mathrm{X}} \square$ and ${ }^{\mathrm{Y}} \mathrm{Li}^{\mathrm{Y}} \mathrm{Al}^{\mathrm{Y}} \mathrm{Al}-{ }^{\mathrm{X}} \square$. As a consequence, weak peaks at 3630,3644 and $3671 \mathrm{~cm}^{-1}$ in the Raman spectrum of primary schorl-type tourmaline Tur IB can be interpreted as caused by, respectively, ${ }^{\mathrm{Y}} \mathrm{Fe}^{* 2+{ }^{\mathrm{Y}}} \mathrm{Al}^{\mathrm{Y}} \mathrm{Al}^{-}{ }^{\mathrm{X}} \square$, ${ }^{\mathrm{Y}} \mathrm{Fe}^{* 2+\mathrm{Y}} \mathrm{Fe}^{* 2+\mathrm{Y}} \mathrm{Al}-{ }^{\mathrm{X}} \square$ and ${ }^{\mathrm{Y}} \mathrm{Li}^{\mathrm{Y}} \mathrm{Fe}^{* 2+\mathrm{Y}} \mathrm{Al}-{ }^{\mathrm{X}} \square$ arrangements, as was proposed by Watenphul et al. [44]. However, the bands are almost invisible in the spectrum of Zn-bearing fluor-elbaite Tur IIIA, although the EMPA data suggest that the $X$ site is not fully occupied in this tourmaline (Tables 3 and 4 ). We interpret it as an indication that the $W$ site in the arrangements is essentially occupied by fluorine. The very weak band at $3676 \mathrm{~cm}^{-1}$, related mainly to the ${ }^{\mathrm{Y}} \mathrm{Li}^{\mathrm{Y}} \mathrm{Fe}^{* 2+\mathrm{Y}} \mathrm{Al}-{ }^{\mathrm{X}} \square$ arrangement, is the only one of this group to appear in the spectrum of Zn-rich fluor-elbaite Tur IIIA. It can easily be explained by the fact that the three cations are the main Y-constituents in this tourmaline.

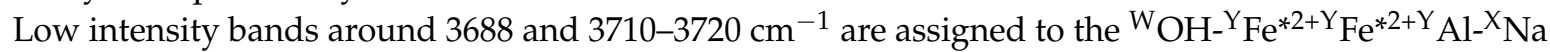
and ${ }^{\mathrm{W}} \mathrm{OH}-{ }^{\mathrm{Y}} \mathrm{Fe}^{* 2+\mathrm{Y}} \mathrm{Fe}^{* 2+\mathrm{Y}} \mathrm{Fe}^{* 2+}{ }^{\mathrm{X}} \mathrm{Na}$ arrangements, whereas an additional band at $3556 \mathrm{~cm}^{-1}$ in the spectrum of $\mathrm{Zn}$-rich fluor-elbaite Tur III A is related to vibrations of ${ }^{\mathrm{W}} \mathrm{OH}$ group, probably bound with some Al-dominant YYY arrangements associated with Na-occupied X sites.

\section{Discussion: Is Natural Zn-Dominant Tourmaline Possible?}

The Zn-rich tourmaline from the Julianna system of anatectic pegmatites at Piława Górna is fluor-elbaite (Tur IIIA) and elbaite (Tur IIIB) with a composition close to $\mathrm{Na}(\mathrm{LiAlZn}) \mathrm{Al}_{6} \mathrm{~B}_{3} \mathrm{Si}_{6} \mathrm{O}_{27}(\mathrm{OH})_{3} \mathrm{~F}$, where $\mathrm{Zn}$ can be partly replaced by $\mathrm{Fe}^{2+}$ and $\mathrm{Mn}^{2+}(\mathrm{Zn}>>\mathrm{Fe}>\mathrm{Mn})$. Sokolov et al. [16], described a Zn-bearing tourmaline with a $\mathrm{Zn}$ content about half of that measured in the Piława Górna tourmaline, and suggested the possible end-member composition $\left.\mathrm{NaZn}_{3} \mathrm{Al}_{6} \mathrm{~B}_{3} \mathrm{Si}_{6} \mathrm{O}_{27}(\mathrm{OH})_{3} \mathrm{OH}\right)$ for a hypothetical Zn-tourmaline. However, two other hypothetical end-members are also possible: $\square\left(\mathrm{Zn}_{2} \mathrm{Al}\right) \mathrm{Al}_{6} \mathrm{~B}_{3} \mathrm{Si}_{6} \mathrm{O}_{27}(\mathrm{OH})_{3}(\mathrm{OH})$ and $\mathrm{Na}\left(\mathrm{Zn}_{2} \mathrm{Al}\right) \mathrm{Al}_{6} \mathrm{~B}_{3} \mathrm{Si}_{6} \mathrm{O}_{27}(\mathrm{OH})_{3} \mathrm{O}_{4}$ by analogy with other tourmalines with divalent Y-occupants, such as schorl/foitite/oxy-schorl and dravite/magnesio-foitite/oxy-dravite. In order to discuss the possibility of the existence of natural Zn-tourmaline two issues should be considered: (1) whether $\mathrm{Zn}$ alone or $\mathrm{Zn}+0.5 \mathrm{Al}$ could dominate the $\mathrm{Y}$ site; and (2) which anion might be expected to be dominant at the $\mathrm{W}$ site of this tourmaline.

Zinc is only a very subordinate incompatible component of silicate magmas, and its lithophile affinity is restricted by the presence of sulphide species. Geochemical fractionation leads to the enrichment of granitic melts, particularly peralkaline magmas, in $\mathrm{Zn}$ and favors crystallization of gahnite as a minor accessory mineral in peraluminous leucogranites, pegmatitic granites and granitic pegmatites, as well as incorporation of traces of Zn into crystal structures of some primary phosphates, e.g., triphylite, lithiophilite, sarcopside or graftonite-group minerals [48]. Lithophile behavior of Zn in the Piława Górna pegmatitic system is evidenced by the crystallization of accessory Zn-bearing ilmenite-pyrophanite and gahnite in moderately fractionated dykes, and the assemblage of gahnite, Zn-bearing ferronigerite and zinconigerite, as well as genthelvite and Zn-bearing helvine in highly-fractionated ones.

In tourmaline-supergroup minerals, $\mathrm{Zn}$ is an accessory component commonly occurring in amounts of up to a few tenths of $\mathrm{ZnO} w \mathrm{t} \%$. Such low concentrations indicate that Zn only slightly fractionates versus Fe in the tourmaline structure. In the Piława Górna pegmatites, even tourmalines from highly-fractionated dykes only occasionally have more than 1 wt \% ZnO. This fact suggests that hypothetical $\mathrm{Zn}$-dominant tourmaline will rather not form due to $\mathrm{Zn}$-Fe fractionation even in geochemically highly-evolved environments. Thus, it seems that the only specific mechanism that 
can promote crystallization of $\mathrm{Zn}$-rich tourmaline is $\mathrm{Na}-\mathrm{Li}$-B-F metasomatism of a gahnite-bearing mineral assemblage. The Zn-rich fluor-elbaite + elbaite (Tur III) from Piława Górna are an example, where the local crystallization environment was controlled by dissolution of gahnite by aggressive $(\mathrm{Na}, \mathrm{Li}, \mathrm{F}, \mathrm{B})$-bearing fluids. Crystallization of $\mathrm{Zn}$-rich tourmaline was, however, limited to a relatively narrow 0.1-millimetre-sized zone around gahnite, which suggests low diffusion of $\mathrm{Zn}$ toward fluor-elbaite overgrowths. Therefore, single crystals of $\mathrm{Zn}$-dominant tourmaline seem rather unlikely in nature and intergrowths of gahnite with Li-bearing tourmalines (Na-Li-B-F metasomatism around gahnite) might be the most favorable environment where zones/domains of $\mathrm{Zn}$-dominant tourmaline could form.

All known tourmalines with elevated $\mathrm{Zn}$ contents are elbaite or fluor-elbaite ([10-16]; present study). The excesses of $\mathrm{Al}$ over $\mathrm{Li}$ and high amounts of $\mathrm{X}$-site vacancies or ${ }^{\mathrm{W}} \mathrm{O}^{2-}$ could favor the formation of $\mathrm{Zn}$-tourmaline with dominance of the end-members $\square\left(\mathrm{Zn}_{2} \mathrm{Al}\right) \mathrm{Al}_{6} \mathrm{~B}_{3} \mathrm{Si}_{6} \mathrm{O}_{27}(\mathrm{OH})_{3}(\mathrm{OH})$ or $\mathrm{Na}\left(\mathrm{Zn}_{2} \mathrm{Al}\right) \mathrm{Al}_{6} \mathrm{~B}_{3} \mathrm{Si}_{6} \mathrm{O}_{27}(\mathrm{OH})_{3} \mathrm{O}$. However, $\mathrm{Zn}$-rich fluor-elbaite with the presence of significant $X$-site vacancies or ${ }^{\mathrm{W}} \mathrm{O}^{2-}$ could also be interpreted as a solid solution of $\mathrm{NaZn}_{3} \mathrm{Al}_{6} \mathrm{~B}_{3} \mathrm{Si}_{6} \mathrm{O}_{27}(\mathrm{OH})_{3}(\mathrm{OH})$ tourmaline with fluor-elbaite + rossmanite or darrelhenryite end-members, and such a case is shown by the Raman spectrum of Zn-rich fluor-elbaite Tur IIIA (the absences of $\mathrm{OH}$ vibration bands, which would originate from ${ }^{\mathrm{Y}} \mathrm{Zn}^{\mathrm{Y}} \mathrm{Al}^{\mathrm{Y}} \mathrm{Al}-{ }^{\mathrm{X}} \square,{ }^{\mathrm{Y}} \mathrm{Zn}^{\mathrm{Y}} \mathrm{Zn}^{\mathrm{Y}} \mathrm{Al}-{ }^{\mathrm{X}} \square$ arrangements). This implies that fluorine, as a significant component of the $\mathrm{Zn}$-rich tourmalines, should be intimately related to the fluor-elbaite constituent, and the $\mathrm{W}$ site in $\mathrm{Zn}$-dominant tourmaline should rather be dominated by $\mathrm{OH}$ or $\mathrm{O}$.

\section{Conclusions}

Atypically high $\mathrm{Zn}$ enrichment in tourmaline is generally connected with certain elbaites and fluor-elbaites. The enrichment in $\mathrm{Zn}$ does not result from $\mathrm{Zn}-\mathrm{Fe}$ fractionation, but rather from dissolution of gahnite by $(\mathrm{Na}, \mathrm{Li}, \mathrm{F}, \mathrm{B}, \mathrm{Be})$-bearing fluid and reprecipitation of $\mathrm{Zn}$-bearing secondary fluor-elbaite or elbaite in the nearest neighborhood of the decomposing gahnite. As fluorine is incorporated into tourmaline by the fluor-elbaite component, three potential $\mathrm{Zn}$-dominant end-members for $\mathrm{Zn}$-tourmaline are possible: $\mathrm{NaZn}_{3} \mathrm{Al}_{6} \mathrm{~B}_{3} \mathrm{Si}_{6} \mathrm{O}_{27}(\mathrm{OH})_{3}(\mathrm{OH})$, $\square\left(\mathrm{Zn}_{2} \mathrm{Al}\right) \mathrm{Al}_{6} \mathrm{~B}_{3} \mathrm{Si}_{6} \mathrm{O}_{27}(\mathrm{OH})_{3}(\mathrm{OH})$ and $\mathrm{Na}\left(\mathrm{Zn}_{2} \mathrm{Al}\right) \mathrm{Al}_{6} \mathrm{~B}_{3} \mathrm{Si}_{6} \mathrm{O}_{27}(\mathrm{OH})_{3} \mathrm{O}$. Future structural studies are needed to determine which of the hypothetical end-members is more likely.

Acknowledgments: We thank three anonymous reviewers for their comments on this manuscript. The studies were supported by the National Science Centre (Poland) grant 2015/19/B/ST10/01809 to AP.

Author Contributions: A.P. designed the scientific topic of the work, made all compositional recalculations and wrote the paper; A.W. and E.S. performed the EMP analysis; B.G. and P.J. recorded and resolved Raman spectra of the tourmalines; and A.S. edited text of the manuscript and prepared graphs.

Conflicts of Interest: The authors declare no conflict of interest. The founding sponsors had no role in the design of the study; in the collection, analyses, or interpretation of data; in the writing of the manuscript, and in the decision to publish the results.

\section{References}

1. Henry, D.J.; Novák, M.; Hawthorne, F.C.; Ertl, A.; Dutrow, B.L.; Uher, P.; Pezzotta, F. Nomenclature of the tourmaline-supergroup minerals. Am. Mineral. 2011, 96, 895-913. [CrossRef]

2. Sokolov, M.; Martin, R.F. A Pb-dominant member of the tourmaline group, Minh Tien granitic pegmatite, Luc Yen District, Vietnam. Estudos Geol. 2009, 19, 352-353.

3. Lottermoser, B.G.; Plimer, I.R. Chemical variation in tourmalines, Umberatana, South Australia. N. Jahrb. Mineral. Mon. 1987, 7, 314-326.

4. Yu, J.M.; Jiang, S.Y. Chemical composition of tourmaline from the Yunlong tin deposit, Yunnan, China: Implications for ore genesis and mineral exploration. Miner. Petrol. 2003, 77, 67-84. [CrossRef]

5. Johnson, M.L.; Wentzell, C.Y.; Elen, S. Multicolored bismuth-bearing tourmaline from Lundazi, Zambia. Gems Gemol. 1997, 33, 204-211. [CrossRef] 
6. Vereshchagin, O.S.; Rozhdestvenskaya, I.V.; Frank-Kamenetskaya, O.V.; Zolotarev, A.A.; Mashkovtsev, R.I. Crystal chemistry of Cu-bearing tourmalines. Am. Mineral. 2013, 98, 1610-1616. [CrossRef]

7. Baksheev, I.A.; Kudryavtseva, O.E. Nickeloan tourmaline from the Berezovskoe gold deposit, Middle Urals, Russia. Can. Mineral. 2004, 42, 1065-1078. [CrossRef]

8. Van Hinsberg, V.J.; Henry, D.J.; Dutrow, B.L. Tourmaline as a petrologic forensic mineral: A unique recorder of its geologic past. Elements 2011, 7, 327-332. [CrossRef]

9. Grice, J.D.; Ercit, T.S. Ordering of Fe and $\mathrm{Mg}$ in the tourmaline crystal structure: The correct formula. N. Jahrb. Miner. Abh. 1993, 165, 245-266.

10. Federico, M.; Andreozzi, G.B.; Lucchesi, S.; Graziani, G. Compositional variation of tourmaline in the granitic pegmatite dykes of the Cruzeiro Mine, Minas Gerais, Brazil. Can. Mineral. 1998, 36, 415-431.

11. Hawkins, K.D.; MacKinnon, I.D.R.; Schneeberger, H. Influence of chemistry on the pyroelectric effect in tourmaline. Am. Mineral. 1995, 80, 491-501. [CrossRef]

12. Adusumilli, M.S.; Castro, C.; Bhaskara Rao, A. Blue and green gem tourmalines from Gregório pegmatite, Rio Grande do Norte State, Brazil. In Proceedings of the 16th General Meeting, IMA, Pisa, Italy, 4-9 September 1994; pp. 1-13.

13. Soares, D.R. Contribuição à Petrologia de Pegmatitos Mineralizados em Elementos Raros e em Elbeítas Gemológicas da Província Pegmatítica da Borborema, Nordeste do Brasil. Ph.D. Thesis, UFPE, Recife, Brazil, 2004.

14. Ferreira, A.C.M.; Ferreira, V.P.; Soares, D.R.; Vilarroel-Leo, H.S. Chemical and mineralogical characterization of elbaite from the Alto Quixaba pegmatite, Seridó Province, NE Brazil. An. Acad. Bras. Ciênc. 2005, 77, 729-743. [CrossRef] [PubMed]

15. Jedwab, J. Tourmaline zincifere dans une pegmatite de Muika (Congo). Bull. Soc. Belge Géol. Paléontol. D'hydrol. 1962, 71, 132-135.

16. Sokolov, P.B.; Gorskaya, M.G.; Kretser, Y.L. Zinc-bearing tourmalines from rare-metal pegmatites. Zap. Vses. Mineral. Obshchest. 1988, 117, 70-74. (In Russian)

17. Szuszkiewicz, A.; Szełęg, E.; Pieczka, A.; Ilnicki, S.; Nejbert, K.; Turniak, K.; Banach, M.; Łodziński, M.; Różniak, R.; Michałowski, P. The Julianna pegmatite vein system at the Piława Górna mine, Góry Sowie Block, SW Poland-preliminary data on geology and descriptive mineralogy. Geol. Q. 2013, 57, 467-484. [CrossRef]

18. Pieczka, A.; Łodziński, M.; Szełeg, E.; Ilnicki, S.; Nejbert, K.; Szuszkiewicz, A.; Turniak, K.; Banach, M.; Michałowski, P.; Różniak, R. The Sowie Mts. pegmatites (Lower Silesia, SW Poland): A current knowledge. Acta Mineral. Petrogr. Szeged 2012, 7, 105-106.

19. Pieczka, A.; Szuszkiewicz, A.; Szełęg, E.; Nejbert, K.; Łodziński, M.; Ilnicki, S.; Turniak, K.; Banach, M.; Hołub, W.; Michałowski, P.; et al. (Fe,Mn)-(Ti,Sn)-(Nb,Ta) oxide assemblage in a little fractionated portion of a mixed (NYF + LCT) pegmatite from Piława Górna, the Sowie Mts. block, SW Poland. J. Geosci. 2013, 58, 91-112. [CrossRef]

20. Pieczka, A.; Szuszkiewicz, A.; Szełeg, E.; Janeczek, J.; Nejbert, K. Granitic pegmatites of the Polish part of the Sudetes (NE Bohemian massif, SW Poland). In Fieldtrip Guidebook, Proceedings of the 7th International Symposium on Granitic Pegmatites, PEG 2015, Ksiąz, Poland, $17-19$ June 2015; Gadas, P., Novák, M., Szuszkiewicz, A., Cempírek, J., Eds.; Czech Republic: Brno, Czech Republic, 2015; pp. 73-103.

21. Pieczka, A.; Szuszkiewicz, A.; Szełęg, E.; Ilnicki, S.; Nejbert, K.; Turniak, K. Samarskite-group minerals and alteration products: An example from the Julianna pegmatitic system, Piława Górna, SW Poland. Can. Mineral. 2014, 52, 303-319. [CrossRef]

22. Pieczka, A.; Szełęg, E.; Szuszkiewicz, A.; Gołębiowska, B.; Zelek, S.; Ilnicki, S.; Nejbert, K.; Turniak, K. Cs-bearing beryl evolving to pezzottaite from the Julianna pegmatitic system, SW Poland. Can. Mineral. 2016, 54, 115-124. [CrossRef]

23. Szuszkiewicz, A.; Pieczka, A.; Szełę, E.; Turniak, K.; Ilnicki, S.; Nejbert, K. The euxenite-group minerals and products of their alteration in the hybrid Julianna granitic pegmatite, Piława Górna, Sudetes, southwestern Poland. Can. Mineral. 2016, 54, 879-898. [CrossRef]

24. Pieczka, A.; Hawthorne, F.C.; Cooper, M.A.; Szełęg, E.; Szuszkiewicz, A.; Turniak, K.; Nejbert, K.; Ilnicki, S. Pilawite-(Y), $\mathrm{Ca}_{2}(\mathrm{Y}, \mathrm{Yb})_{2}\left[\mathrm{Al}_{4}\left(\mathrm{SiO}_{4}\right)_{4} \mathrm{O}_{2}(\mathrm{OH})_{2}\right]$, a new mineral from the Piława Górna granitic pegmatite, southwestern Poland: Mineralogical data, crystal structure and association. Mineral. Mag. 2015, 79, 1143-1157. [CrossRef] 
25. Szełęg, E.; Zuzens, B.; Hawthorne, F.C.; Pieczka, A.; Szuszkiewicz, A.; Turniak, K.; Nejbert, K.; Ilnicki, S.S.; Friis, H.; Makovicky, E.; et al. Bohseite, ideally $\mathrm{Ca}_{4} \mathrm{Be}_{4} \mathrm{Si}_{9} \mathrm{O}_{24}(\mathrm{OH})_{4}$, from the Piława Górna quarry, the Góry Sowie Block, SW Poland. Mineral. Mag. 2017, 81, 35-46. [CrossRef]

26. Pieczka, A.; Hawthorne, F.C.; Ma, C.; Rossman, G.; Szełeg, E.; Szuszkiewicz, A.; Turniak, K.; Nejbert, K.; Ilnicki, S.S. Żabińskiite, ideally $\mathrm{Ca}\left(\mathrm{Al}_{0.5} \mathrm{Ta}_{0.5}\right)\left(\mathrm{SiO}_{4}\right) \mathrm{O}$, a new mineral of the titanite group from the Piława Górna pegmatite, the Góry Sowie Block, southwestern Poland. Mineral. Mag. 2017, 81, 591-610. [CrossRef]

27. Turniak, K.; Pieczka, A.; Kennedy, A.K.; Szełęg, E.; Ilnicki, S.; Nejbert, K.; Szuszkiewicz, A. Crystallisation age of the Julianna pegmatite system (Góry Sowie Block, NE margin of the Bohemian massif): Evidence from U-Th-Pb SHRIMP monazite and CHIME uraninite studies. In Fieldtrip Guidebook, Proceedings of the 7th International Symposium on Granitic Pegmatites, PEG 201, Ksią̇, Poland, 17-19 June 2015; Gadas, P., Novák, M., Szuszkiewicz, A., Cempírek, J., Eds.; Czech Republic: Brno, Czech Republic, 2015; pp. 111-112.

28. Van Breemen, O.; Bowes, D.R.; Aftalion, M.; Żelaźniewicz, A. Devonian tectonothermal activity in the Sowie Góry gneissic block, Sudetes, southwestern Poland: Evidence from $\mathrm{Rb}-\mathrm{Sr}$ and $\mathrm{U}-\mathrm{Pb}$ isotopic studies. Ann. Geol. Soc. Pol. 1988, 58, 3-10.

29. Timmermann, H.; Parrish, R.R.; Noble, S.R.; Kryza, R. New U-Pb monazite and zircon data from the Sudetes Mountains in SW Poland: Evidence for a single-cycle Variscan orogeny. J. Geol. Soc. 2000, 157, 265-268. [CrossRef]

30. Kryza, R.; Fanning, C.M. Devonian deep-crustal metamorphism and exhumation in the Variscan Orogen: Evidence from SHRIMP zircon ages from the HT-HP granulites and migmatites of the Góry Sowie (Polish Sudetes). Geodin. Acta 2007, 20, 159-176. [CrossRef]

31. Müller, A.; Szuszkiewicz, A.; Ilnicki, S.; Nejbert, K. Quartz chemistry of the Julianna pegmatites and their wall rocks, Piława Górna, Poland: Implications for the origin of pegmatite melts. In Abstracts, Proceedings of the 8th International Symposium on Granitic Pegmatites, PEG2017, Kristiansand, Norway, 13-15 June 2017; Müller, A., Rosing-Schow, N., Eds.; Norsk Geologisk Forening: Trondheim, Norway, 2017; pp. 84-88.

32. Pouchou, I.L.; Pichoir, F. "PAP" (phi-rho-z) procedure for improved quantitative microanalysis. In Microbeam Analysis; Armstrong, I.T., Ed.; San Francisco Press: San Francisco, CA, USA, 1985; pp. 104-106.

33. Pesquera, A.; Gil-Crespo, P.P.; Torres-Ruiz, F.; Torres-Ruiz, J.; Roda-Robles, E. A multiple regression method for estimating $\mathrm{Li}$ in tourmaline from electron microprobe analyses. Mineral. Mag. 2016, 80, 1129-1133. [CrossRef]

34. SPECTRA-CALC V2.23. Galactic Industries Corp.: Main St., NH, USA.

35. Whitney, D.L.; Evans, B.W. Abbreviations for names of rock-forming minerals. Am. Mineral. 2010, 95, 185-187. [CrossRef]

36. Alvarez, M.A.; Coy-Yli, R. Raman spectra of tourmaline. Spectrochim. Acta A 1978, 34, 899-908. [CrossRef]

37. Gasharova, B.; Mihailova, B.; Konstantinov, L. Raman spectra of various types of tourmalines. Eur. J. Mineral. 1997, 9, 935-940. [CrossRef]

38. McKeown, D.A. Raman spectroscopy, vibrational analysis, and heating of buergerite tourmaline. Phys. Chem. Miner. 2008, 35, 259-270. [CrossRef]

39. Hoang, L.H.; Hien, N.T.M.; Chen, X.B.; Minh, N.V.; Yang, I.-S. Raman spectroscopis study of various types of tourmalines. J. Raman Spectrosc. 2011, 42, 1443-1446. [CrossRef]

40. Skoggby, H.; Bosi, F.; Lazor, P. Short-range order in tourmaline: A vibrational spectroscopic approach to elbaite. Phys. Chem. Miner. 2012, 39, 811-816. [CrossRef]

41. Zhao, C.; Liao, L.; Xia, Z.; Sun, X. Temperature-dependent Raman and infrared spectroscopy study on iron-magnesium tourmalines with different Fe content. Vib. Spec. 2012, 62, 28-34. [CrossRef]

42. Fantini, C.; Tavares, M.C.; Krambrock, K.; Moreira, R.L.; Righi, A. Raman and infrared study of hydroxyl sites in natural uvite, fluor-uvite, magnesio-foitite, dravite and elbaite tourmalines. Phys. Chem. Miner. 2014, 41, 247-254. [CrossRef]

43. Berryman, E.J.; Wunder, B.; Ertl, A.; Koch-Müller, M.; Rhede, D.; Scheidl, K.; Giester, G.; Heinrich, W. Influence of the X-site composition tourmaline's crystal structure: Investigation of synthetic K-dravite, dravite, oxy-uvite, and magnesio-foitite using SREF and Raman spectroscopy. Phys. Chem. Miner. 2016, 43, 83-102. [CrossRef]

44. Watenphul, A.; Burgdorf, M.; Schlüter, J.; Horn, I.; Malcherek, T.; Mihailova, B. Exploring the potential of Raman spectroscopy for crystallochemical analyses of complex hydrous silicates: II. Tourmalines. Am. Mineral. 2016, 101, 970-985. [CrossRef] 
45. Mercurio, M.; Rossi, M.; Izzo, F.; Cappelletti, P.; Germinario, C.; Grifa, C.; Petrelli, M.; Vergara, A.; Langella, A. The characterization of natural gemstones using non-invasive FT-IR spectroscopy: New data on tourmalines. Talanta 2018, 178, 147-159. [CrossRef] [PubMed]

46. Choi, J.B.; Hawthorne, F.C. Characterization of tourmaline crystals by Rietveld and single-crystal structure refinement: A comparative study. Geosci. J. 2002, 6, 237-243. [CrossRef]

47. Hawthorne, F.C. Structural mechanism for light-element variations in tourmaline. Can. Mineral. 1996, 34, 123-132.

48. Černý, P.; Meintzer, R.E.; Anderson, A.J. Extreme fractionation in rare-element granitic pegmatites: Selected examples of data and mechanism. Can. Mineral. 1985, 23, 381-421.

2018 by the authors. Licensee MDPI, Basel, Switzerland. This article is an open access article distributed under the terms and conditions of the Creative Commons Attribution (CC BY) license (http://creativecommons.org/licenses/by/4.0/). 